See discussions, stats, and author profiles for this publication at: https://www.researchgate.net/publication/330660892

\title{
Integrated European intra-day electricity market: Rules, modeling and analysis
}

Article in Applied Energy · March 2019

Dol: 10.1016/.j.apenergy.2018.12.073

\section{CITATIONS}

3 authors:

Le Hong Lam

University of Science and Technology - The University of Danang

11 PUBLICATIONS 44 CITATIONS

SEE PROFILE

Cristian Bovo

Politecnico di Milano

97 PUBLICATIONS 703 CITATIONS

SEE PROFILE

Some of the authors of this publication are also working on these related projects:

Project INGRID View project

European Electricity Market View project
115

Ilea Valentin

Politecnico di Milano

35 PUBliCATIONS 165 CITATIONS

SEE PROFILE 


\title{
Integrated European Intra-Day Electricity Market: Rules, Modeling and Analysis
}

\author{
Le Hong Lam ${ }^{\mathrm{a}, \mathrm{b}, *}$, Valentin Ilea ${ }^{\mathrm{a}}$, Cristian Bovo ${ }^{\mathrm{a}}$ \\ ${ }^{a}$ Department of Energy, Politecnico di Milano, Via La Masa, 34, 20156 Milano, Italy \\ ${ }^{b}$ Faculty of Electrical Engineering, University of Science and Technology - The University of Danang, Danang, Vietnam
}

\begin{abstract}
Currently, the coupling of the European Electricity Markets has been fully achieved for the Day-Ahead Market. In the same time, a joint integrated Intra-Day Market based on the Continuous Trading mechanism is under implementation and it involves countries from West of Europe. However, some countries (e.g. Italy and Iberian countries) use the Discrete Auction mechanism and their integration implies the harmonization of the two trading mechanisms. A Hybrid Mechanism that represents the coordination of Continuous Trading and Discrete Auction has been discussed with two concrete proposals being made by Italy and Iberian countries. Thus, the aim of this paper is to propose an advanced algorithm which can simulate the clearing of the integrated European Intra-Day Market with any levels of coordination between Discrete Auction and Continuous Trading. The proposed model is formulated as a Mixed Integer Linear Problem and incorporates all market rules of both mechanisms. The algorithm is flexible enough to adapt to future changes. In addition, a case study that considers the current and near future market participants and covers an entire day of an Intra-Day Market is run by an iterative process to investigate the impact of the hybrid Intra-Day Market model on the Iberian countries and Italy according to their proposals. Moreover, the test cases represent realistic data in terms of number of variables and constraints, and in terms of complexity of the bids. The results of the paper show that, on one hand, the proposed market clearing model can be applied for different levels of Continuous Trading and Discrete Auction coordination and, on the other hand, the integration into the single European Intra-Day Market of Italy and Iberian countries can be beneficial for them. Therefore, the general conclusion is that the proposed market clearing model can be successfully used by the Power Exchanges currently integrated to solve the market or by the Power Exchanges not yet integrated to evaluate the impact of their future integration. On the other hand, the model can easily cope with any further changes in the market rules and represents a tool for future research, e.g. analysis of market participants behavior in future scenarios or evaluation of various financial instruments to manage the congestion of intra-border interconnectors.
\end{abstract}

Keywords: XBID project, European Intra-Day Market, Continuous Trading, Discrete Auction.

\section{Highlights}

- proposes a model achieving complete integration of European Intra-Day Market rules.

- the model can sustain any level of integration of markets with different rules.

- the results confirm the near-future developments of the actual market.

- the building block for evaluating the future evolution of the market is provided.

\section{Acronyms}

AEP Average Electricity Price

AON All-Or-Nothing

\footnotetext{
* Corresponding author

Email address: honglam.le@polimi.it (Le Hong Lam)
}

ATC Available Transfer Capacity

BO Block Order

CTMA Continuous Trading Matching Algorithm

CT Continuous Trading

CRA Complementary Regional Auction

DA Discrete Auction

DAM Day-Ahead Market

DPM Data Processing Module

FOK Fill-Or-Kill

GCT Gate Closure Time

GFS Good-For-Session

GTD Good-Till-Date

IOC Immediate-Or-Cancel 
IO

Iceberg Order

IDM Intra-Day Market

MO Multi-period Order

MSO Market Sweep Order

OM Optimization Module

PX Power Exchange

RES Renewable Energy Source

SOB Share Order Book

SO Single period Order

SW Social Welfare

TS Trading Session

TSO Transmission System Operator

TV Trading Volume

VWAP Volume Weighted Average Price

\section{Nomenclature}

\section{Sets}

$a \in A \quad$ Set of bidding areas.

$a \in A_{p} \quad$ Set of bidding areas participating in the current CT session, where $A_{p} \subseteq A$.

$t \in T \quad$ Set of periods, including $h \in T^{h}$ (set of hourly periods), $h h \in T_{h}^{h h}$ (set of half-hourly periods), $q \in T_{h h}^{q}$ (set of quarter-hourly periods), with $t=(h, h h, q), T=T^{h} \cup T_{h}^{h h} \cup$ $T_{h h}^{q}$. Here, each hour $h$ includes two halfhours $h h$ and each half hour includes two quarter-hours $q$.

$t \in T_{p} \quad$ Set of periods considered in the current CT session, where $T_{p} \subseteq T$.

Sets of Interconnectors

$l \in B r_{l} \quad$ Set of interconnectors including $B r_{l}^{A C}$ and $B r_{l}^{D C}$ (the set of AC and DC interconnectors), respectively; where $B r_{l}=B r_{l}^{A C} \cup$ $B r_{l}^{D C}$. Element $l$ is defined by connected areas $f a \in A$ and $t a \in A$. The power flow is positively defined from $f a$ to $t a$.

$l \in B r_{l}^{p} \quad$ Subset of interconnectors considered in the current CT session, where $B r_{l}^{p}=B r_{l}^{A C, p} \cup$ $B r_{l}^{D C, p}, B r_{l}^{p} \subseteq B r_{l}$.

$l s \in L S \quad$ Set of all line sets $l s$. $l \in l s \quad$ Set of interconnectors subject to the same group constraint.

\section{Sets of Orders}

$s \in S_{a} \quad$ Set of single period orders in area $a$, in which $S_{a}^{s}$ is the subset of supply orders and $S_{a}^{d}$ is the subset of demand orders, with $S_{a}=$ $S_{a}^{s} \cup S_{a}^{d}$.

$i o \in I O_{a} \quad$ Set of iceberg orders in area $a$, where $I O_{a} \subseteq$ $S_{a}$.

$i c \in i o \quad$ Set of clips belonging to an iceberg order $i o$.

$l k \in L K_{a} \quad$ Set of linked orders in area $a$, where $L K_{a} \subseteq$ $S_{a}$.

$m \in M_{a} \quad$ Set of multi-period orders in area $a$, where $M_{a}=M S_{a} \cup B_{a}$.

$b \in B_{a} \quad$ Set of block orders in area $a$.

$m s \in M S_{a} \quad$ Set of market sweep orders in area $a$, in which $M S_{a}^{s}$ is the subset of supply orders and $M S_{a}^{d}$ is the subset of demand orders, with $M S_{a}=M S_{a}^{s} \cup M S_{a}^{d}$.

\section{Parameters}

The value of submitted quantity $Q$ is positive for demand side and negative for supply side.

$R_{l / l s}^{t, U}, R_{l / l s}^{t, D} \quad$ Hourly ramping energy up and down of single interconnector or line set in period $t$, in $\mathrm{MWh} / \mathrm{h}$, where $R_{l / l s}^{t, U} \in \Re^{+} ; R_{l / l s}^{t, D} \in \Re^{-}$.

$B_{i o}^{i c, t} \quad$ Binary parameter indicating the accepted status of iceberg clip $i c$ in period $t$, it equals 1 if the iceberg clip is fully accepted, 0 otherwise.

$B r_{t}^{l, \max / \min }$ Maximum and minimum capacity of interconector $l$ in MWh.

Penality coefficient for interconnector $l$ in $€ /$ MWh.

$f_{l}^{t, \text { int }} \quad$ The power flow of previous CT in interconnector $l$, equals zero for the first $\mathrm{CT}$.

$I M_{i o}^{i c} \quad$ Incidence matrix of iceberg order $i o$ and iceberg clip $i c$. Thus, the $k m-t h$ element of $I M_{i o}^{i c}$ is 1 if $k \in i o$ is iceberg order in the $i c$ clip.

$I M_{s}^{l k} \quad$ Incidence matrix of link order $l k$ and parent orders lo. Thus, the $k m-t h$ element of $I M_{s}^{l k}$ is 1 if $k \in s$ is the parent of $l k \in L K_{a}$, otherwise is null. 
$\operatorname{loss}_{l} \quad$ Losses coefficient of interconnector $l$ in per-
centages with respect to the transmitted power.

$Q_{m}, P_{m} \quad$ Submitted quantity and price of multiple period order, in MWh and $€ / M W h$, respectively.

$Q_{s}^{t}, P_{s}^{t} \quad$ Submitted quantity and price of single period order in dispatch period $t$, in MWh and $€ /$ MWh, respectively.

$R_{s}^{\min }, R_{m}^{\min } \quad$ The minimum accepted ratio of order $s, m$, in p.u.

$T_{m}^{s}, T_{m}^{e} \quad$ Starting and ending period of multiple period order $m$, respectively.

$T_{s}^{i}, T_{m}^{i} \quad$ Incoming time of single period order $s$, multiple period order $m$ in minutes since continuous trading has started.

$T_{s}^{r}, T_{m}^{r} \quad$ Removing time of single period order $s$, multiple period order $m$ in minutes since continuous trading has started.

$U_{m}^{t, s}, U_{m}^{t, e} \quad$ Starting and ending unit step of multiple period order $m$ in dispatch period $t$. Here, its value is equal to 1 if $t \geq T_{m}^{s}\left(>T_{m}^{e}\right)$ an 0 otherwise.

\section{Continuous Variables}

$x_{s}^{t}, x_{m}^{t}$

Accepted ratio of single $s$ and multi $m$ periods order.

$P_{a}^{t} \quad$ Net position of area $a$ in period $t$ in MWh.

$f_{l}^{t} \quad$ Power flow in interconnector $l$ in period $t$ in MWh, where $l \in \Re$.

$f_{l}^{t,+/-} \quad$ Positive and negative power flow in interconnector $l$ in period $t$ in MWh, where $f_{l}^{t,+} \in$ $\Re^{+}$and $f_{l}^{t,-} \in \Re^{-}$.

\section{Binary Variables}

$Y_{m}$

Status of multi-period order $m$, it equals 1 if accepted.

$Y_{s}^{t}$

Status of single period order $s$ in period $t$, it equals 1 if accepted.

\section{Introduction}

The European Directive 2009/28/EC [1] mandated that, by $2020,20 \%$ of the EU final energy consumption should be produced from Renewable Energy Sources (RESs) while $10 \%$ of the European Union energy consumption in transport should be produced from RESs. Generally, this has the positive effect of having a drastic reduction of pollution levels. However, the high share of RES, such as the wind and solar energy, also negatively impacts on the power system operation: the inherent fluctuations of RES increase the power imbalance of the system. Traditionally, at national level, one way to mitigate this is by using a short-term, close to time of dispatch electricity market: the Intra-Day Market (IDM). This market allows the adjustment of the quantities cleared in the Day-Ahead Market (DAM) to a profile closer to the real time operation.

Already, the DAM in Europe is operated as a single integrated market where the following Power Exchanges (PXs) participate [2]: APX (Netherlands and United Kingdom), Belpex (Belgium), EPEX Spot (France, Germany and Austria), GME (Italy), Nordpool Spot (Scandinavian and Baltic Countries), OMIE (Iberian), OPCOM (Romania), TGE (Poland), and OTE (Czech Republic). The operation of the European DAM is showing numerous benefits, e.g for GME [3].

In turn, a guideline on Capacity Allocation and Congestion Management for the European IDM was established on $24^{\text {th }}$ July, 2015 [4] and it represented the first step towards the integrated European IDM.

Moreover, the European Commission has established an European Target Model to achieve the Internal Energy Market in which the Cross-Border Intra-Day Market Project (abbreviated XBID project) is representative for the European IDM [5], and is based on the Continuous Trading (CT) mechanism and implicit auction [6]. Here, market participants submitting the orders in one country can be matched by orders submitted in any other countries as long as transmission capacity is available and they are remunerated using the pay-as-bid mechanism.

In order to operate the integrated IDM in Europe, a proposal developed by all Transmission System Operators (TSOs) and PXs is presented in [7]. As in [6], the operation of integrated IDM is based on the Continuous Trading market design, and the market clearing algorithm is called the Continuous Trading Matching Algorithm (CTMA). The CTMA, executed for each Trading Session (TS), contains three major steps: (i) maximize economic surplus, (ii) update the submitted bids database, known as Share Order Book (SOB), and (iii) re-calculate available capacity. In order to ensure the fair and orderly price formation for all products, the price-time-priority is also adopted and the requirements of CTMA are detailed in [8]. Here, as in the other documents $[5,6,7]$, only the specifications of CTMA are given in general terms, while the actual mathematical model is not shown.

Until the beginning of 2018, the PXs already operating in the integrated European IDM were EPEX Spot, Nordpool Spot, Belpex, APX. Particularly, Switzerland is a part of this project while it does not participate in the integrated European DAM project. Moreover, up to this point, the European IDM was operated in CT but this has become complex as there are countries (e.g. Italy and Iberian countries) which have a national IDM operated in Discrete Auctions (DAs), i.e similarly to the DAM but with a shorter time horizon. Thus, the integration of these 
countries would require that they shift to the CT mechanism, but this is very difficult to achieve in a short to medium time horizon due to the dramatic change in the regulatory framework it would require. Therefore, a hybrid market structure where both mechanisms coexists is the solution to fully achieve the integrated European IDM in the short-time horizon. In fact, Italy and Iberian countries, both operating in DAs based national IDM, proposed in 2017 two hybrid mechanism $[9,10]$ for their integration into the European IDM: (i) one very close to their national IDM structure, for a fast integration, and (ii) one closer to the CT mechanism, for a fuller integration. Since June $12^{\text {th }} 2018$, the Iberian countries are integrated using the first option [11], while Italy is still not integrated. However, up to now, no official document has discussed the hybrid market structure in terms of mathematical model nor the impact of the hybrid market structure has been analyzed, e.g. the effects of the two options proposed by Italy and Iberian countries.

The up-to-date research dealing with the coupling of IDM in Europe is mostly related to statistical analysis of the historical data and treats poorly the modeling of the CTMA. These research papers can be arranged into two major categories: (i) papers investigating the CT based structure of IDM [12, 13, 14, 15, 16, 17] and (ii) papers forecasting possible scenarios for the future European IDM $[18,19,20]$.

The articles [12] and [13] analyze deeply about the market behaviors in CT for the ELBAS ${ }^{1}$ market and for Spanish market using historical data. These papers state that the RES penetration impacts significantly on the bidding strategy of the players: due to the DA in Spain, the players will bid such that a high balancing reserve will be required while the players in ELBAS prefer submitting bids close to the delivering time because of the operation of CT. An interesting paper published by Hagemann and Weber in [14] presents an analytical model to investigate the effect of stochastic sources on the liquidity of IDM using market data of 2012 and 2013. This paper draws two main conclusions: (i) in France and Denmark only the biggest companies can trade in $\mathrm{CT}$, while the players in Germany are more homogeneous and (ii) the design of DA is not the cause of the high trading volume in the market but the market peculiarities are.

Papers [15] and [16] investigate the impact of quarterlyhour offers in both DA and CT of the German IDM. Paper [15] analyses the result of the implementation of the quarterly-hour offer in DA, while paper [16] investigates the impact of quarterly-hour offers through the 15 minutes electricity price in CT on the bidding behavior of RES plants. These papers show clear evidence that the behavior of the market is improved because of the implementation of quarterly hour offers, with a great increment

${ }^{1}$ ELBAS is the IDM formed by all Scandinavian and Baltic countries. of Trading Volume (TV), a growth of flexibility in the market and a smoothed electricity price. Once again, paper [17] emphasizes the important role of IDM in mitigating the imbalance risk exposure of RESs using the German IDM, however, this paper is limited by a domestic view, an approximated exchange of energies between countries, and a simplified forecast error modeling.

Paper [18] estimates the gaining from the market integration using the extrapolating ACER's partial coverage methodology and the real data from 2012 to 2014. Here, the benefit of the single market is very clear since it adds $1.3 \mathrm{bn} € /$ year in the IDM and balancing market. At the same time, the authors indicate two major issues: (i) a non-sufficient capacity of interconnectors and (ii) the urgency to find a shared solution for balancing and reserve. Paper [19] presents a set of policy recommendations for the future electricity market design considering the high share of RESs in Europe, in line with the European target. Here, two principles which support the continuous trading mechanism of the integrated European IDM are Principle 1-correct the market failures as close as possible to their source, relying on subsidiarity as much as possible and Principle 2-allow for appropriate cross-country variation in market design across European Member States rather than a one-size-fits-all-solution.

In general, the tools used to perform the analysis in the above papers, e.g. the forecasting models in $[14,17]$ or statistic models in $[15,16,18]$, are different in nature with respect to a market clearing algorithm. They require such an algorithm to represent the market rules, but they use very simplified ones.

According to the well-informed knowledge of the authors, there is only one work, [20], which is related to the CTMA. This paper only proposes the automatic matching process to update the remaining capacity of interconnectors in each TS and it does not consider the complexity of CTMA when it comes to modeling various types of bids present in IDM. Moreover, the DA mechanism and its coordination with the CT are not considered. The paper concludes that the CT can sensibly increase the welfare. In particular, this conclusion is derived from a simple example which is not compatible with the complexity of the European market topology, thus it is too far when compared to the full requirements of the single European IDM $[8]$.

Therefore, as of today, there is no research that proposes a complete and detailed mathematical model for solving the integrated European IDM. Last but not least, the reader may argue that in literature there are many papers dealing with market clearing algorithms, e.g [21, 22, $23]$ to name a few. However, these papers deal with the DAM which is a very different approach with respect to the IDM. With particular reference to the European case, the DAM model is solved only one time per day and its main challenge is the non-convexity of the bids due to the market rules of block orders, Minimum Income Condition orders, National Uniform Price orders. Meanwhile, the 
European IDM model is dealing with different characteristics in terms of market designs and market rules, i.e. solving the market dynamically and the DA-CTcoordination. In detail, the non-convexity of block orders is dealt in the IDM by introducing the pay-as-bid mechanism in CT and by not considering some types of bids, like the National Uniform Price orders. Moreover, particular types of bids not present in the DAM are considered in the IDM, like the Market Sweep Orders, along with specific CT restrictions, namely the execution and the validity restrictions. These, together with more stringent computation time requirements (10 minutes for DAM [24] against a few seconds for CT [8]) determine the use of a different approach to model the European IDM.

It is clear from the previous paragraphs that there is a complete lack of information regarding the mathematical representation of the market clearing model for the European IDM, similar to the EUPHEMIA [21, 25] for the European DAM. Moreover, such representation is mandatory to correctly analyze various aspects of the market, such as market player behavior dynamics or the impact of the evolution of the generation sources. For these reasons, the aim of this paper is to:

- Propose an advanced optimization model which can simulate the DA, CT and the DA-CT coordination containing all the market rules (order types, network constraints, etc.) encountered across Europe and able to cope with the future integration of DA market areas into the single European IDM and, in general, the evolution of the market;

- Investigate the impact of various hybrid mechanisms (e.g. [9] and [10]) on the operation of the single European IDM using a realistic study case in terms of number of bids, type of bids and network, that represents the near-future evolution of the market.

The paper is organized as follows: Section 2 describes the current IDM in Europe, Section 3 presents the mathematical model. Next, Section 4 not only presents the detail of two proposals [9] and [10] but also proposes an iterative process to run a whole length of IDM in one day. Then, the results and their discussions are shown in Section 5. Finally, the relevant conclusions are illustrated in Section 6.

\section{State-of-the-art of the Intra-Day Market in Eu- rope}

\subsection{Market design}

In Europe, two IDM mechanisms are currently used by PXs: CT and DA. The GME and OMIE use DA, while the rest of the European PXs rely on CT, except Germany who uses both.

The CT means that the trade can be settled whenever the offer arrives (through a TS) and the price-time priority rule is applied: if the submitted price of orders is the same, the priority is given to the earliest submitted order. The latest time before a TS for players to submit the bids is the Gate Closure Time (GCT) that is defined by PXs, e.g 30 minutes for France, Germany, Austria, 5 minutes for Belgium and Netherlands, 60 minutes for Switzerland and for cross-border exchange [26], 45 minutes for ELBAS, 20 minutes for exchanges between ELBAS and Germany [27]. The bid which can not be executed has the possibility to enter the SOB where all available bids are located and wait for the next TS. The time validity of a TS. i.e. the time window for which the clearing is performed, is defined by the market operator. In general, for $\mathrm{CT}$, the time validity of the TS has its upper limit at the end of the day [14], while the lower limit is given by the GCT.

The DA is cleared by the marginal pricing rule in several auctions held at fixed points in time: seven for $\mathrm{GME}^{2}$ [28], six for $\mathrm{OMIE}^{3}[29]$ and one for Germany [30].

The major advantage of CT [12] is that it allows market players to trade whenever they can as long as the trading capacity of interconnectors is sufficient; it thus minimizes the expected energy imbalance in a very short time-frame. Meanwhile, the DA is characterized by a large distance from closing time to delivery time, but it has higher TV than that in CT [13].

Obviously, the different designs of IDMs in Europe means that harmonizing them into a single European IDM is a major challenge: each design has its own advantages and is very difficult for the PXs to perform market rules changes. Therefore, as with the European DAM, the European IDM will be a unified platform of heterogeneous market rules rather than a homogeneous market with simple rules.

\subsection{Type of orders}

Orders greatly differ between various PXs. According to [7], two major types of orders can be distinguished: (i) Single period Order (SO) and (ii) Multi-period Order (MO). SOs are defined for only one period such as hourly, half-hourly, quarter-hourly, meanwhile, MOs are defined for several consecutive hourly periods. The orders can be entered without any execution restriction (official acronym: NON [31, 7]) or with only one execution restriction such as Fill-Or-Kill (FOK), Immediate-Or-Cancel (IOC) and All-Or-Nothing (AON), and together with or without the validity restrictions: Good-For-Session (GFS) or Good-Till-Date (GTD).

Table 1 resumes the major differences between the execution restrictions in terms of accepted quantity and possibility to enter SOB if the order can not be matched in the current TS. The order which can not be executed and can not enter the SOB will not appear in the next TS.

\footnotetext{
${ }^{2}$ Each DA of GME is denoted from MI1 to MI7

${ }^{3}$ Each DA of OMIE is denoted from S01 to S06
} 
Table 1: The execution restrictions in European IDM

\begin{tabular}{ccccc}
\hline \multirow{2}{*}{ Restrictions } & \multicolumn{2}{c}{ Accepted } & \multicolumn{2}{c}{ SOB } \\
\cline { 2 - 5 } & Fully & Partially & Enter & Delete \\
\hline NON & - & $\checkmark$ & $\checkmark$ & - \\
\hline FOK & $\checkmark$ & - & - & $\checkmark$ \\
\hline IOC & - & $\checkmark$ & - & $\checkmark$ \\
\hline AON & $\checkmark$ & - & $\checkmark$ & - \\
\hline
\end{tabular}

Here, it should be noted that SOs following link order rule (which has a linked execution constraint [21, 31]) always contain FOK restriction. SOs are called an Iceberg Order (IO) if they contain a large volume which is divided into several smaller orders, called iceberg clips, that enter the market sequentially only if the previous clip in the sequence was already fully accepted. The volume for every clip is the same, meanwhile, the price can be the same with the first clip or incremental/decremental with respect to the previous clip.

On the other hand, MOs entered with AON restriction is called Block Orders (BOs). Meanwhile, MOs entered with IOC are called Market Sweep Orders (MSOs). The MSOs are particular orders as they can only be matched with SOs already in SOB; they can not be matched with any other MOs [31].

Finally, the main difference between the validity restrictions is that the market player can define the time until the submitted bid remain in the SOB, which means that the GTD restriction is imposed; or can choose to let the submitted bid stay in the SOB as long as the time validity of the order is entirely covered by the current or future TSs, which means that the GFS restriction is imposed. Finally, the validity restrictions are set only for the orders with NON or AON restriction as they are the only ones that can remain in the SOB for future TSs.

\subsection{Capacity allocation management}

Today, the capacity allocation management in the European DAM uses the hybrid model which contains both Available Transfer Capacity (ATC) and Flow-Based models [25]. Meanwhile, the IDMs in Europe only rely on the ATC model. Thus, the available capacity for the first TS of IDM in the Flow-Based coupling region is re-calculated and adapted to the ATC model by the TSOs [32]. At this moment, the X-BID project supports both explicit (where decided by National Regulatory Authorities) and implicit capacity allocation to allocate cross-border capacity to users continuously. The number of interconnectors using the explicit allocation has been reduced from three to one (Germany-France) since 16th of June, 2018 [33]. The explicit allocation is a standalone capacity request by users entities, while the implicit allocation is triggered by trades generated in SOB. Thus, while the explicit alloca- tion is an independent trade, similar to a bilateral transaction, the implicit allocation is a consequence of the market clearing algorithm. For this reason, the limits of the interconnectors used in implicit allocation already include the effect of the explicit allocation. Therefore, the paper only simulates the implicit continuous allocation method.

\section{Mathematical model}

The structure of the integrated European IDM algorithm contains two main building blocks. The basic one (named TS Matching Model) consists in finding the market solution for a given TS while the second one (named Transition TS Model) models the transit from one TS to the next. The second building block is described in Section 4.2, while the basic one is detailed here. The proposed TS Matching model presented in Fig. 1 contains two main modules: (i) Optimization Module (OM) and (ii) Data Processing Module (DPM). For each TS of either CT or DA session, the OM maximizes the economic surplus considering all bids (new submitted bids and bids in the SOB) and satisfying all economic and network constraints. Meanwhile, the DPM is responsible for elaborating the SOB and the ATC of interconnectors for the next TS.

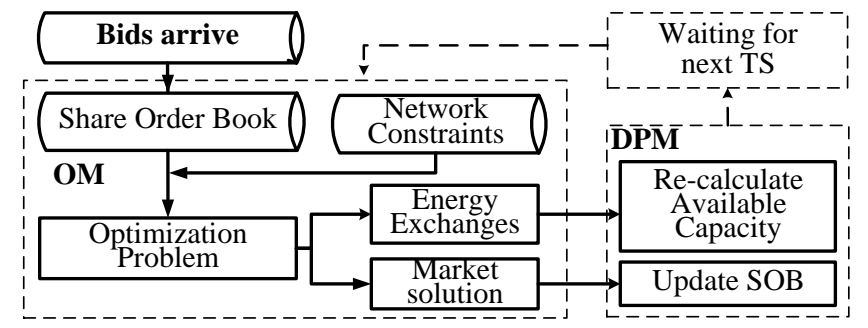

Figure 1: Trading Matching model for one TS

\subsection{Optimization Model}

First of all, it should be noted that this model can be used to run DA, CT, or the coordination of DA-CT. In order to achieve this, the set of participant bidding areas $A_{p}$ and the set of considered periods in current TS, $T_{p}$ are properly set (hence, $T^{h} \subseteq T_{p}, T_{h}^{h h} \subseteq T_{p}$ and $T_{h h}^{q} \subseteq T_{p}$ ); before the optimization problem is solved.

\subsubsection{Objective function}

$$
\operatorname{Max}\left[\sum_{a \in A_{p}} \sum_{t \in T_{p}}\left(c_{a}^{t, s u r}-c_{a}^{t, p r}\right)-\sum_{t \in T_{h h}^{q}} \sum_{l \in B r_{l}^{p}} c_{l}^{t, p e n}\right]
$$

Equation (1) comprises three terms: (i) the economic surplus of demand and supply, $c_{a}^{t, s u r}$; (ii) a penalty term modeling the price-time priority rule, $c_{a}^{t, p r}$ and (iii) a penalty term related to the decomposition of power flow into positive and negative power flow to model the losses of DC interconnectors, $c_{l}^{p e n}$. It should be noted that the term (ii) is not required for the DA, but only for CT. 
As with any conventional market, the goal of this model (1) is to maximize the Social Welfare (SW):

$$
c_{a}^{t, s u r}=\sum_{s \in S_{a}} Q_{s}^{t} \cdot x_{s}^{t} \cdot P_{s}^{t}+\sum_{m \in M_{a}} Q_{m} \cdot x_{m}^{t} \cdot P_{m}
$$

Moreover, a penalty term $c^{t, p r}$ is defined to model the price-time priority rule:

$$
c_{a}^{t, p r}=\left(\sum_{s \in S_{a}}\left|Q_{s}^{t}\right| \cdot x_{s}^{t} \cdot T_{s}^{i}+\sum_{m \in M_{a}}\left|Q_{m}\right| \cdot x_{m}^{t} \cdot T_{m}^{i}\right) \cdot \varepsilon
$$

Notably, in CT sessions, a time priority rule is applied for the bids that have the same submitted price: priority is given to the earliest submitted bid. Mathematically, the price-time priority rule considers only marginal bids that have the same submitted price. Thus, the welfare created by (2) is constant with respect to the incoming time of the bids, while the penalty term (3) is forcing a loss of welfare for the latest submitted bids. In this way, priority is given to the execution of the bid which has the lowest value of incoming time $\left(T_{s}^{i}\right.$ and $\left.T_{m}^{i}\right)$. Moreover, indirectly, this mechanism assures the unicity of the solution by distinguishing between bids with equal submitted price. To guaranty the maximization of SW, the parameter $\varepsilon$ is added in (3) and set to very small value (e.g 0.0001). So that, the value of $\mathrm{SW}$, i.e. $c_{a}^{t, p r}$ is not perturbed significantly.

The total SW is also penalized by the term $c_{l}^{t, p e n}$. This term, defined by equation (4), has the main role to correctly decompose the power flows in all interconnectors $\left(f_{l}^{t}\right.$ and $\left.f_{l, m s}^{t}\right)$ into complementary positive $\left(f_{l}^{t,+}\right.$ and $\left.f_{l, m s}^{t,+}\right)$ and negative $\left(f_{l}^{t,-}\right.$ and $\left.f_{l, m s}^{t,-}\right)$ power flows. This means that when $f_{l}^{t,+} / f_{l, m s}^{t,+}$ are non-null, $f_{l}^{t,-} / f_{l, m s}^{t,-}$ need to be null and vice-versa. This decomposition is required to correctly model the real energy losses on such interconnectors [34] and limit the upper and lower bounds of temporary variable $f_{l, m s}^{t}$. Therefore, $c_{l}$ for all interconnectors are set to 0.001 . This parameter is set such that the associated penalty term does not have a negative impact on the market solution, i.e. such that minimizing the power flow of interconnectors instead of maximizing the SW is avoided. Moreover, this term has a secondary role: it guaranties an optimal distribution of the market areas net positions into flows on the meshed ATC interconnectors optimizing, thus, the exploitation of their capacities. This penalty term is given by:

$$
c_{l}^{t, p e n}=c_{l} \cdot\left(f_{l}^{t,+}-f_{l}^{t,-}+f_{l, m s}^{t,+}-f_{l, m s}^{t,-}\right)
$$

In (4), variables $f_{l, m s}^{t,+}$ and $f_{l, m s}^{t,-}$ are related to the MSOs and are explained in detail latter in the next subsection.

\subsubsection{Balancing equations}

$$
\begin{aligned}
& \left(\sum_{s \in S_{a}} Q_{s}^{h} \cdot x_{s}^{h}\right) / 4+\left(\sum_{s \in S_{a}} Q_{s}^{h h} \cdot x_{s}^{h h}\right) / 2+\left(\sum_{s \in S_{a}} Q_{s}^{q} \cdot x_{s}^{q}\right) \\
& +\left(\sum_{m \in M_{a}} Q_{m} \cdot x_{m}^{t}\right) / 4-P_{a}^{q}=0 \\
& \forall h \in T^{h}, h h \in T_{h}^{h h}, q \in T_{h h}^{q}, a \in A_{p}
\end{aligned}
$$

$$
\begin{aligned}
& P_{a}^{t}+\sum_{l \in B r_{f a . a}^{A C, p}} f_{l}^{t}+\sum_{l \in B r_{f a . a}^{D C, p}}\left(f_{l}^{t,+} \cdot\left(1+l o s s_{l}\right)+f_{l}^{t,-}\right) \\
& -\sum_{l \in B r_{a . t a}^{A C, p}} f_{l}^{t}-\sum_{l \in B r_{a . t a}^{D C, p}}\left(f_{l}^{t,+}+f_{l}^{t,-} \cdot\left(1+\operatorname{loss}_{l}\right)\right)=0 \\
& \forall t \in T_{h h}^{q}, a \in A_{p} \\
& f_{l}^{t,+}-B r_{l, \text { max }}^{t} / 4 \leq 0 \quad \forall l \in B r_{l}^{p}, t \in T_{h h}^{q} \\
& B r_{l, m i n}^{t} / 4-f_{l}^{t,-} \leq 0 \quad \forall l \in B r_{l}^{p}, t \in T_{h h}^{q} \\
& f_{l}^{t}-f_{l}^{t,+}+f_{l}^{t,-}=0 \\
& \forall l \in B r_{l}^{p}, t \in T_{h h}^{q}
\end{aligned}
$$

Equality constraint (5) defines the net position of area $a, P_{a}^{t}$, in quarterly-hour $q$. Thus, the energy of hourly orders is divided by 4 and half-hourly orders is divided by 2. Consequently, it allows various production orders in different areas to match together, enhancing thus the liquidity of the market. Meanwhile, equality constraint (6) ensures that the net positions are balanced by the power transits at each area. The loss coefficient $\operatorname{loss}_{l}, f_{l}^{t,+}$ and $f_{l}^{t,-}$ are added in (6) to take into account the losses in the DC interconnectors and decompose the power flow into importing and exporting. Constraints (7)-(9) combined with the penalty term (4) give $f_{l}^{t,+}$ and $f_{l}^{t,-}$ as in [34].

\subsubsection{Order constraints}

Inequality constraints (10)-(13) model all market rules for SOs. Inequality constraint (10)-(11) represent the executed restrictions of SOs without IOs. The accepted ratio of SOs $\left(x_{s}^{t}\right)$ is in the range $\left[R_{\text {min }}^{s}, 1\right]$. Thus, for orders subject to fully accepted or rejected restriction (FOK and AON) $R_{\min }$ is equal to 1 , while is equal to 0 for partially accepted restriction (NON and IOC). The constraint of link orders is shown in (12) where link order is fully accepted or rejected according to the value of the binary variable $Y_{s}$. The relationship between the link orders is qualified by the matrix $I M_{s}^{l k}$ :

$$
\begin{aligned}
& Y_{s}^{t} \cdot R_{s}^{\text {min }}-x_{s}^{t} \leq 0 \\
& \forall s \in S_{a} \backslash I O_{a}, t \in T_{p}, a \in A_{p} \\
& x_{s}^{t}-Y_{s}^{t} \leq 0 \\
& \forall s \in S_{a} \backslash I O_{a}, t \in T_{p}, a \in A_{p} \\
& x_{l k}^{t}-\sum_{s \in S_{a}} I M_{s}^{l k} \cdot Y_{s}^{t} \leq 0 \\
& \forall l k \in L K_{a}, t \in T_{p}, a \in A_{p}
\end{aligned}
$$

Inequality constraint (13), written for each iceberg clip $i c$, ensures that $i c \in i o$ is executed sequentially. Here, the first clip is not considered because the first element of $I M_{i o}^{i c}$ is null. The initial value of parameter $B_{i o}^{i c, t}$ is null and updated in DPM:

$$
\begin{aligned}
& x_{i c}^{t} \cdot I M_{i o}^{i c}-B_{i o}^{i c-1, t} \leq 0 \\
& \forall i c \in i o, i o \in I O_{a}, t \in T_{p}, a \in A_{p}
\end{aligned}
$$

It should be pointed out that the DA only contains hourly SOs with IOC restriction, meanwhile the CT includes all types of orders and with different product orders 
such as hourly, half-hourly and quarterly-hour orders. The constraints for different product orders are modelled similarly and distinguished by the sets $T^{h} \subseteq T_{p}, T_{h}^{h h} \subseteq T_{p}$ and $T_{h h}^{q} \subseteq T_{p}$.

Constraints (14)-(23) model the market rules of MOs that include BOs (14)-(15) and MSOs (14)-(23). The BOs are always subject to AON restriction, thus $R_{m}^{m i n}$ equals to 1 and $Y_{m}$ is a free variable. When (14)-(15) model the BOs, the accepted ratio $x_{m}^{t}$ is in the range $\left[Y_{m}, Y_{m} \cdot\left(U_{m}^{t, s}-\right.\right.$ $\left.\left.U_{m}^{t, e}\right)\right]$.

The MSOs are subjected to IOC restriction, thus $R_{m}^{\min }$ of MSOs equals to 0 and the binary variable $\left(Y_{m}\right)$ in $(14)-(15)$ is fixed to 1 . When (14)-(15) model the MSOs, the accepted ratio $x_{m}^{t}$ in the range $\left[0,\left(U_{m}^{t, s}-U_{m}^{t, e}\right)\right]$.

Likewise SOs in (10)-(11), constraints (14)-(15) also model the execution restriction of MOs. Since MOs are defined for several consecutive periods, the term of unit step $\left(U_{m}^{t, s}-U_{m}^{t, e}\right)([21,34])$ is added in (14)-(15) to set up the upper bound of the accepted ratio $x_{m}^{t}$. If it is inside the submitted periods (from $T_{m}^{s}$ to $T_{m}^{e}$ ): $x_{m}^{t}$ of one $\mathrm{BO}$ is forced to be equal to 1 or 0 for the entire period, meanwhile, $x_{m}^{t}$ of one MSOs is in the range [0,1]; otherwise $x_{m}^{t}$ is equal to 0 :

$$
\begin{aligned}
& Y_{m} \cdot R_{m}^{m i n}-x_{m}^{t} \cdot\left(U_{m}^{t, s}-U_{m}^{t, e}\right) \leq 0 \\
& \forall m \in M_{a}, t \in T^{h}, a \in A_{p} \\
& x_{m}^{t}-Y_{m} \cdot\left(U_{m}^{t, s}-U_{m}^{t, e}\right) \leq 0 \\
& \forall m \in M_{a}, t \in T^{h}, a \in A_{p}
\end{aligned}
$$

The previous constraints (5)-(15) alone do not guarantee the satisfaction of the clearing rule of the MSO orders, i.e. MSOs can only be matched with SOs. For this, it is necessary for the total accepted volume of the MSOs supply or demand, respectively, to only be cleared by a fraction of the total accepted volume of the SOs demand or supply, respectively, while satisfying market constraints. Conceptually, this means that the MSOs need to be also cleared separately from the other MOs in an "independent" market session where only SOs and MSOs are present. However, this "independent" market needs to be properly linked to the actual market session constraints (5)-(13) in order to achieve correct market results.

From a mathematical standpoint, this is achived by introducing the intermediate variables, $P_{a}^{q, m s}, f_{l, m s}^{t}, f_{l, m s}^{t,+}$, $f_{l, m s}^{t,-}, x_{s}^{t, m s}, Y_{s}^{t, m s}$ and $Y_{m}^{m s}$, to represent the "independent" MSO market session and link it to the main one. Then constraints (16)-(20) are introduced to model this "independent" session; here, (17) and (20) repeat the balancing equations (6), (9) and market rules of SOs (10)-(13), respectively, but all original variables are replaced by the intermediate ones. The intermediate net position $P_{a}^{q, m s}$ is defined in (16) as a function of only MSOs (the $x_{m}^{t}$ variables) and the fraction of SOs they match (the $x_{s}^{t, m s}$ ). This fraction needs to be lower, at maximum equal, to the actual cleared volume of the SOs (the $x_{s}^{t}$ variables) which is achieved by the inequality constraint (21). Then, since the MSOs and SOs are also present in the main constraints (5)-(13) where they determine the actual power flows on interconnectors $\left(f_{l}^{t}, f_{l}^{t,+}\right.$ and $\left.f_{l}^{t,-}\right)$, and here they also need to be cleared over all the areas, then, the intermediate power flows created here by the MSOs $\left(f_{l, m s}^{t}, f_{l, m s}^{t,+}\right.$ and $\left.f_{l, m s}^{t,-}\right)$ are limited by the actual power flows in the inequality constraints (18) and (19):

$$
\begin{aligned}
& \left(\sum_{s \in S_{a}} Q_{s}^{h} \cdot x_{s}^{h, m s}\right) / 4+\left(\sum_{s \in S_{a}} Q_{s}^{h h} \cdot x_{s}^{h h, m s}\right) / 2 \\
& +\sum_{s \in S_{a}} Q_{s}^{q} \cdot x_{s}^{q, m s}+\left(\sum_{m \in M S_{a}} Q_{m} \cdot x_{m}^{t}\right) / 4-P_{a}^{q, m s}=0 \\
& \forall h \in T^{h}, h h \in T_{h}^{h h}, q \in T_{h h}^{q}, a \in A_{p} \\
& (6),(9) \\
& f_{l, m s}^{t,+}-f_{l}^{t,+} \leq 0 \quad \forall l \in B r_{l}^{p}, t \in T_{h h}^{q} \\
& f_{l}^{t,-}-f_{l, m s}^{t,-} \leq 0 \quad \forall l \in B r_{l}^{p}, t \in T_{h h}^{q} \\
& \text { (10)-(13) } \\
& x_{s}^{t, m s}-x_{s}^{t} \leq 0 \quad \forall s \in S_{a}, t \in T^{h}, a \in A_{p}
\end{aligned}
$$

Inequality constraints (22)-(23) impose that the total accepted volume of MSO demand is not higher than the total accepted volume of SO supply with which it is matched and in reverse for MSO supply and SO demand. Thus, these constraints ensure that MSOs can not be matched with themselves:

$$
\begin{aligned}
& \left(\sum_{m \in M S_{a}^{d}} Q_{m} \cdot x_{m}^{t}\right) / 4+\left(\sum_{s \in S_{a}^{s}} Q_{s}^{h} \cdot x_{s}^{h, m s}\right) / 4 \\
& +\left(\sum_{s \in S_{a}^{s}} Q_{s}^{h h} \cdot x_{s}^{h h, m s}\right) / 2+\left(\sum_{s \in S_{a}^{s}} Q_{s}^{q} \cdot x_{s}^{q, m s}\right) / 4 \leq 0 \\
& \forall h \in T^{h}, h h \in T_{h}^{h h}, q \in T_{h h}^{q}, a \in A_{p} \\
& \left(\sum_{m \in M S_{a}^{s}} Q_{m} \cdot x_{m}^{t}\right) / 4+\left(\sum_{s \in S_{a}^{d}} Q_{s}^{h} \cdot x_{s}^{h, m s}\right) / 4 \\
& +\left(\sum_{s \in S_{a}^{d}} Q_{s}^{h h} \cdot x_{s}^{h h, m s}\right) / 2+\left(\sum_{s \in S_{a}^{d}} Q_{s}^{q} \cdot x_{s}^{q, m s}\right) / 4 \geq 0 \\
& \forall h \in T^{h}, h h \in T_{h}^{h h}, q \in T_{h h}^{q}, a \in A_{p}
\end{aligned}
$$

\subsubsection{Network constraints}

This model only considers the current IDM in Europe, and thus it only contains the ATC inequality constraints (24)-(27), and ramping up and down of exchange energy on single interconnector or group of interconnectors between two consecutive periods (28)-(31).

Inequality constraints (24)-(25) limit the energy flow through the interconnectors by two parameters $B r_{l}^{h, \max }$ and $B r_{l}^{h, \min }$ in each quarterly-hour period. Since $B r_{l}^{h, \max }$

and $B r_{l}^{h, \text { min }}$ are in MWh, as provided by the TSO, they are divided by 4 to provide the boundary of quarterly-hour energy flow:

$$
\begin{array}{ll}
f_{l}^{t}-B r_{l}^{h, \max } / 4 \leq 0 & \forall l \in B r_{l}^{p}, t \in T_{h h}^{q} \\
B r_{l}^{h, \text { min }} / 4-f_{l}^{t} \leq 0 & \forall l \in B r_{l}^{p}, t \in T_{h h}^{q}
\end{array}
$$


Meanwhile, inequality constraints (26)-(27) limit the upper and lower bound of temporary power flow $f_{l, m s}^{t}$ using the actual positive $\left(f_{l}^{t,+}\right)$ and negative $\left(f_{l}^{t,-}\right)$ power flow:

$$
\begin{array}{ll}
f_{l, m s}^{t}-f_{l}^{t,+} \leq 0 & \forall l \in B r_{l}^{p}, t \in T_{h h}^{q} \\
f_{l}^{t,-}-f_{l, m s}^{t} \leq 0 & \forall l \in B r_{l}^{p}, t \in T_{h h}^{q}
\end{array}
$$

Inequality constraints (28)-(31) model the ramping constraints and they limit the variation of exchanged energy between two consecutive periods on individual interconnectors (28)-(29) or group of interconnectors (30)-(31) to reduce risks that might threaten the security of supply due to frequent large changes in the production. If the interconnector is not subject to ramping limit, $R U$ and $R D$ have large values. Here, the total exchanged energy on interconnector is computed for each hour. For the current TS, the energy exchanged in the previous TS is also considered by the parameter $f_{l}^{t-1, i n t}$ in order to compute the actual energy exchanges in the considered TS:

$$
\begin{aligned}
& \sum_{t \in T_{h}^{h h}} \sum_{t \in T_{h h}^{q}}\left(f_{l}^{t}+f_{l}^{t, i n t}\right)-\sum_{t \in T_{h}^{h h}} \sum_{t \in T_{h h}^{q}}\left(f_{l}^{t-1}+f_{l}^{t-1, i n t}\right) \\
& -R_{l}^{t, U} \leq 0 \forall l \in B r_{l}^{p}, t \in T^{h} \\
& \sum_{t \in T_{h}^{h h}} \sum_{t \in T_{h h}^{q}}\left(f_{l}^{t-1}+f_{l}^{t-1, i n t}\right)-\sum_{t \in T_{h}^{h h}} \sum_{t \in T_{h h}^{q}}\left(f_{l}^{t}+f_{l}^{t, i n t}\right) \\
& +R_{l}^{t, D} \leq 0 \forall l \in B r_{l}^{p}, t \in T^{h} \\
& \sum_{l \in l s} \sum_{t \in T_{h}^{h h}} \sum_{t \in T_{h h}^{q}}\left(f_{l}^{t}+f_{l}^{t, i n t}\right)-\sum_{l \in l s} \sum_{t \in T_{h}^{h h}} \sum_{t \in T_{h h}^{q}}\left(f_{l}^{t-1}\right. \\
& \left.+f_{l}^{t-1, i n t}\right)-R_{l s}^{t, U} \leq 0 \forall l s \in L S, t \in T^{h} \\
& \sum_{l \in l s} \sum_{t \in T_{h}^{h h}} \sum_{t \in T_{h h}^{q}}\left(f_{l}^{t-1}+f_{l}^{t-1, i n t}\right)-\sum_{l \in l s} \sum_{t \in T_{h}^{h h}} \sum_{t \in T_{h h}^{q}}\left(f_{l}^{t}\right. \\
& \left.+f_{l}^{t, i n t}\right)+R_{l s}^{t, D} \leq 0 \forall l s \in L S, t \in T^{h}
\end{aligned}
$$

\subsection{Data processing module (DPM)}

According to Fig. 1, the DPM is launched after the solution of the OM has been found. Therefore, at this step, the accepted ratio $\left(x_{s}^{t}\right.$ and $\left.x_{m}^{t}\right)$ and the exchanged energy $\left(f_{l}^{t}\right)$ in the current TS are known. Now, this module will prepare the data for the next TS, acting on:

\subsubsection{Shared order book}

The SOB collects all the bids that were not fully executed $\left(x^{t} \neq 1\right)$ and are subjected to NON or AON restriction. Thus, in the case of partial execution $\left(0<x^{t}<1\right)$, the order will be allocated in SOB with the un-executed volume $\left(Q^{t} \cdot\left(1-x^{t}\right)\right)$ and the initial submitted price $\left(P^{t}\right)$. Moreover, the validity restrictions (GFS and GTD) are checked for the parameters $T_{s}^{r}$ and $T_{m}^{r}$ and the validated orders are deleted: if the current time is later than the $T_{s}^{r}$ and $T_{m}^{r}$, the bid is deleted from the SOB. Finally, the parameter $B_{i o}^{i c}$ of the iceberg order is updated as well. If $i c$ is fully accepted in period $t, B_{i o}^{i c, t}$ equals 1 .

\subsubsection{Available transfer capacity}

In the real life, TSOs are responsible for this activity with a complex procedure to provide $B_{l}^{h, \max }$ and $B_{l}^{h, \min }$ in real time. In this paper, parameter $B_{l}^{h, \max }$ and $B_{l}^{h, \text { min }}$ are updated to $B_{l, \text { new }}^{h, \text { max }}$ and $B_{l, \text { new }}^{h, \text { min }}$ for the next TS according to (32) and (33). This is made to emulate the real time behavior of the ATC limits according to the requirements of CTMA [8]. Thus, according to (32) and (33), they are updated with respect to the sign of $f_{l}^{t}$ : if $f_{l}^{t}$ is positive, $B_{l, \text { new }}^{h, \text { ix }}$ is reduced and $B_{l, \text { new }}^{h, \text { min }}$ is increased; if $f_{l}^{t}$ is negative, $B_{l, \text { new }}^{h, \text { max }}$ is increased and $B_{l, \text { new }}^{h, \text { in }}$ is decreased:

$$
\begin{aligned}
& B_{l, \text { new }}^{h, \text { max }}=B_{l}^{h, \max }-4 \cdot \max _{t \in T_{h h}^{q}}\left(f_{l}^{t}, 0\right)-4 \cdot \min _{t \in T_{h h}^{q}}\left(f_{l}^{t}, 0\right) \\
& \forall l \in B_{l}^{p}, h \in T^{h} \\
& B_{l, \text { new }}^{h, \text { min }}=B_{l}^{h, \text { min }}-4 \cdot \max _{t \in T_{h h}^{q}}\left(f_{l}^{t}, 0\right)-4 \cdot \min _{t \in T_{h h}^{q}}\left(f_{l}^{t}, 0\right) \\
& \forall l \in B_{l}^{p}, h \in T^{h}
\end{aligned}
$$

\section{Hybrid intra-day market mechanism}

\subsection{State-of-the-art}

Until this year, the PXs set to trade with CT (Nordpool Spot, EPEX Spot, APX, Belpex) were coupled together with different GCT to form the integrated European IDM [26]. However, currently a major challenge is how to coordinate the CT running PXs with the incoming PXs running DA, such as GME and OMIE. To answer this question, two official proposals, introduced by GME [9] and OMIE [10], where GCT is 60 minutes have been made: (i) Option A just allows a small degree of coordination preserving the historical design and, thus, it represents a fast way of integration, while (ii) Option B is representative for the new structure with a large degree of coordination. At the present moment, only the IDM of OMIE is coupled through Option A as of June $12^{\text {th }}$ of 2018, but the market design will be changed to Option B in future [11]. In general, due to their very recent or near future integration, the impact of Option $\mathbf{A}$ and Option $\mathbf{B}$ is not yet known. In details:

- Option A in Fig. 2: GME has six DAs (see Fig. 2a) and two of them (MI2 and MI4) are opened to all European PXs using, the so-called Complementary Regional Auction (CRA). OMIE also has six DAs (see Fig. 2b) but there is no CRA. The DA-CT coordination starts from MI2 in Italy and S01 in OMIE and operates as follows: once a DA session is closed a CT session is opened for only periods that are not auctioned anymore. By allowing the DA-CT coordination only to these hours, the level of uncertainties in the capacity calculation is reduced, therefore there is a higher probability of finding additional cross-zonal capacities. 


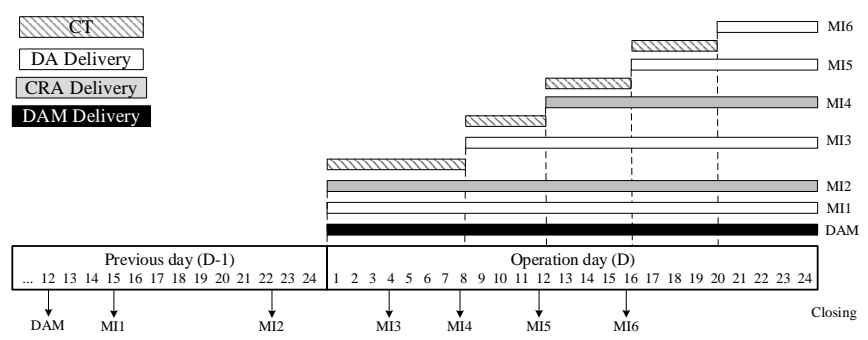

(a)

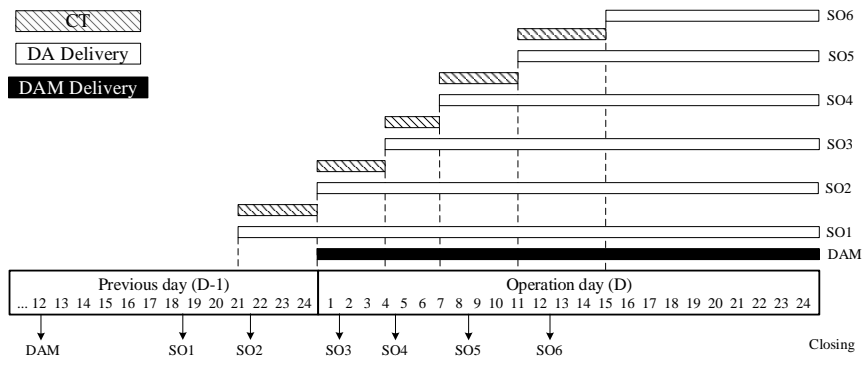

(b)

Figure 2: The Option A: (a) GME and (b) OMIE

In Fig. 2, DAM, CRA and DA delivery stand for the dispatching periods cleared by the DAM and the DA of IDM (MI1-MI6) and (S01-S06) in GME and OMIE, respectively. The CT are periods when GME and OMIE operate in continuous trading mechanism with a GCT of 60 minutes.

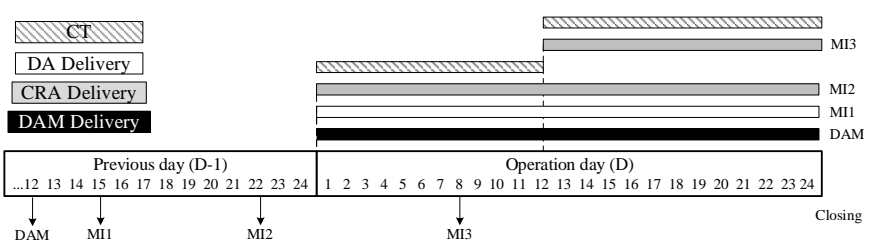

(a)

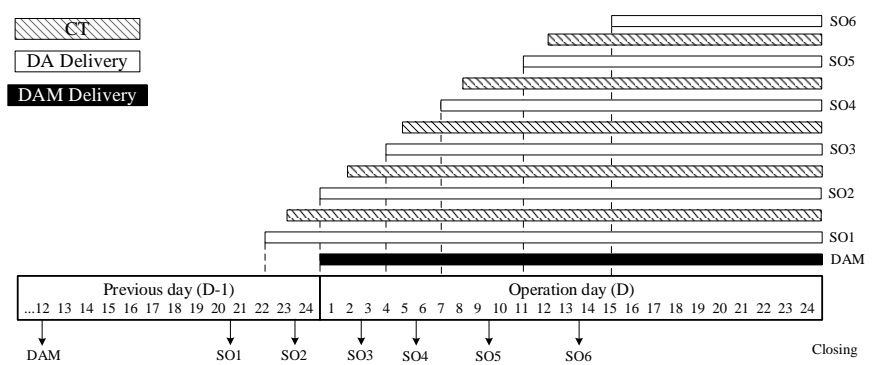

(b)

Figure 3: The Option B: (a) GME and (b) OMIE

- Option B in Fig 3: GME has three DAs (see Fig. 3a), and two are CRAs (MI2 and MI3). OMIE still has six DAs, and no CRA (see Fig. 3b). Here, the DA-CT coordination operates as in Option A for GME starting with MI2. Meanwhile, the coordination in OMIE is wider, in the sense that in any CT session trades can be made for any future period of the same day. It thus enhances the efficiency in using the capacity of interconnectors. As with Option A, the GCT is 60 minutes.

As it can be noticed, in both options OMIE does not open its DAs to the other PXs (it does not have CRAs). This is because, with the Minimum Income Condition adopted in OMIE [29] and the block orders used in CWE, finding the market solution would require too much time, as they introduce non-convex issues and the procedure of clearing the market would be the same with the European DAM [21] taking, thus, even tens of minutes to solve. In fact, the time threshold of executing either DA or CRA is 10 minutes [7], so CRAs are not an option for OMIE.

For both GME and OMIE, the bids in DAs are deleted after solving each DA, then players can modify their production by submitting new bids in the CT session.

\subsection{Transition TS model}

In order to evaluate the impact of the DA-CT coordination, the paper proposes an iterative process in Fig. 4 which simulates the operation of such a market, in which the OM and DPM interact in time; from one TS to the next.

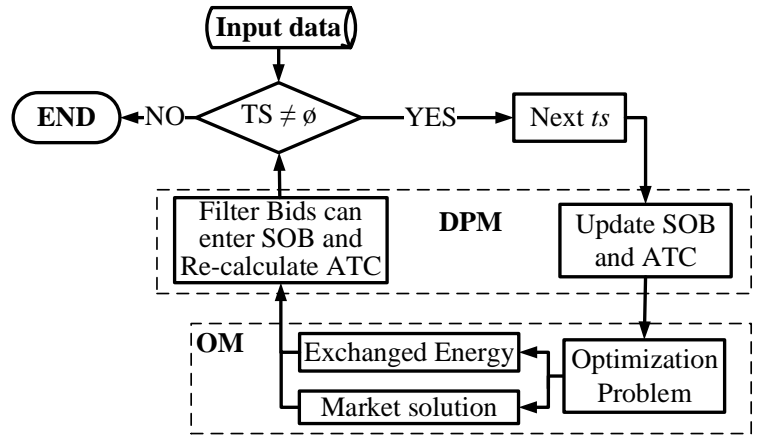

Figure 4: Proposed iterative process

The proposed process contains five main steps:

1. Input data: Initializes the set of $\mathrm{TS}(t s \in T S)$ sequences, in which set $A_{p}$ and $T_{p}$ are created in correspondence to $t s$. The model starts with the first element of $t s \in T S$ and moves to DPM;

2. DPM: With $t s=1$, the SOB receives the initial data corresponding to the first TS. If the $t s \geq 2$, the SOB receives the bids corresponding to the current $t s$ and all the bids that are subject to NON or AON restrictions and were not fully executed in the previous ts (already located in SOB). Moreover, ATC limits are updated;

3. OM: OM receives the data which arrives from DPM and solves the problem defined in Section 3.1 for the current TS. Then, it sends the market results back to DPM; 
4. DPM: As explained in Section 3.2, the un-executed volume of bids that subscribe to NON or AON restriction are computed using the accepted ratio $x$, while if $T_{s}^{r}$ and $T_{m}^{r}$ are smaller than $t s$, the bids are deactivated immediately. Meanwhile, the ATC limits are re-calculated using (32)-(33);

5. Next $t s \in T S$ is activated and second, third and forth steps (DPM and OM) are repeated until the last TS is solved.

In the above process, CT offers are inserted for the corresponding ts (to which they belong) together with the valid bids from SOB. Then, the bids are activated by $T_{s}^{i}$ and $T_{m}^{i}$ (when $T_{s}^{i}$ or $T_{m}^{i}$ are equal $t s$ ) and deactivated by $T_{s}^{r}$ and $T_{m}^{r}$ (when $T_{s}^{r}$ or $T_{m}^{r}$ are smaller than $t s$ ). Additionally, $t s$ can be DA and/or CT, thus the operation of DA and/or CT is decided by the set of $a \in A_{p}$ corresponding to $t s \in T S$.

\section{Test and Results}

\subsection{Case Study}

The case study implements the current configuration of the Integrated European IDM which contains the following PXs: Nordpool Spot (Scandinavian and Baltic countries), EPEX Spot (France, Germany, Switzerland), APX (United Kingdom and Netherlands), Belpex (Belgium), GME (Italy), and OMIE (Iberian countries). The GME is preparing to enter, OMIE is already participating as of June 2018, while the other PXs are integrated for few years. The market topology is shown in Fig. 5 and includes 52 bidding areas and 71 interconnectors. Here, the dark areas belong to GME, the areas with diagonal lines to OMIE while the no-color areas are the CT areas. Germany (DE) uses both market designs. Additionally, the continuous lines show AC interconnectors, while the dashed lines indicate DC interconnectors. The parameters for the network constraints are obtained from the real data of a working day that is Wednesday, $9^{t h}$ of March 2016: the initial ATC limits are obtained from [28] for GME, [29] for OMIE, [35] for EPEX Spot, APX, Belpex and [36] for Nordpool Spot; the interconnectors between CT and DA are obtained from [37]. According to [38], the line-set conditions are applied in bidding area NO1A (NO5-NO1A and NO2-NO1A) and DK1A (NO2-DK1A and SE3-DK1A) of Nord Pool Spot.

The orders of GME and OMIE are publicly available and they were obtained directly from the official websites [28] and [29], respectively. Meanwhile, the orders in Central West of Europe and Northern Europe are not publicly available but only global information is: hourly electricity price, total accepted volume and low resolution graphical files of cumulated curves. Thus, the authors had to create artificially realistic orders using the total hourly (and quarterly) exchanged volume, and maximum, minimum and average prices in [30] for EPEX Spot, APX, Belpex

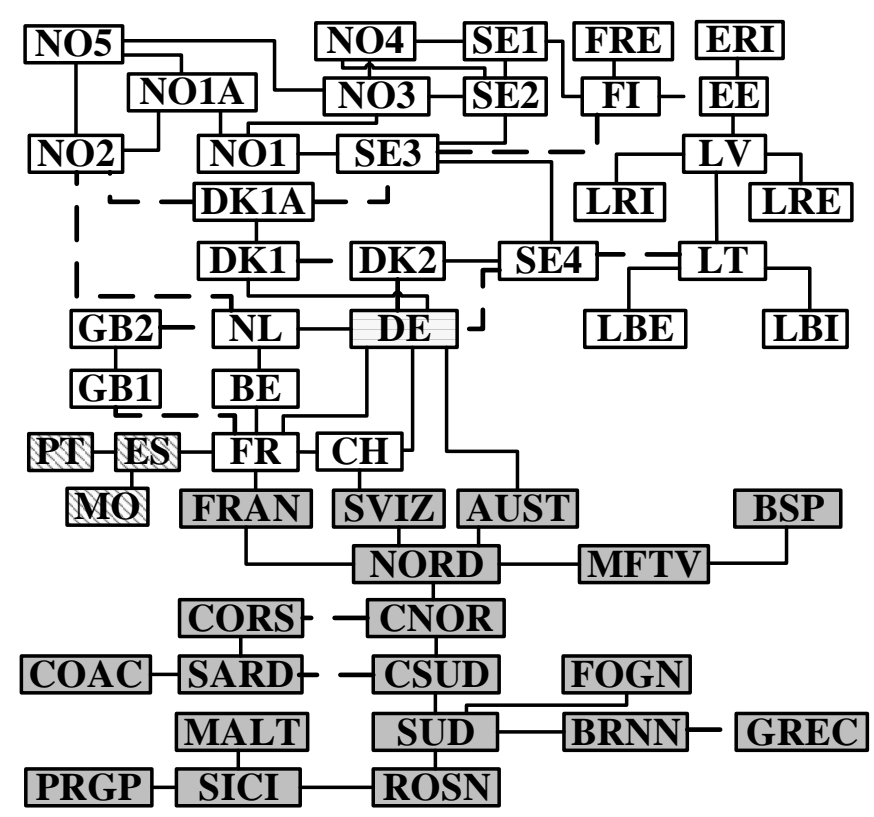

Figure 5: Case study European Intra-day market topology

and [39] for Nord Pool Spot. Here, the total exchange volume for every period $t$ in each area $a$ is split into bids with the same submitted volume; here the number of bids are user-defined such to obtain a realistic number of bids for each type of order. Then, for each of these, the price is chosen randomly in the range [min price, max price]. In particular, for the accepted bids, the price for the demand bids is chosen in the upper half of the range [average price, max price] while the supply bids price in the lower half of the range [min price, average price]. In this way, the accepted volume in the final result of the proposed model will be exactly the same as the real market while the price of accepted bids will be in the real price range.

The resulting number of orders is listed in Table 2. In particular, the data in OMIE considers only the orders of unit producers satisfying the Minimum Income Condition. Moreover, the number of DAs in GME is only five instead of seven because the paper is using the data before GME adopted seven auctions, and was operated with five DAs.

Table 2: The number of orders in the case study

\begin{tabular}{ccc}
\hline Type & Restrictions & Number \\
\hline \multirow{2}{*}{ SO } & NON & 116038 \\
\cline { 2 - 3 } & FOK, IOC, AON & 18364 \\
\hline \multirow{2}{*}{ MO } & AON (BOs) & 161 \\
\cline { 2 - 3 } & IOC (MSOs) & 159 \\
\hline
\end{tabular}

In real life, the CT starts from 14:00 pm of the previous day (D-1) to the last GCT at 10:30 pm of the current day (D) [12] and the GCT for cross-border is 60 minutes between either GME or OMIE and EPEX Spot, while the GCT for local trade is 30 minutes [26]. Therefore, TS 
sequences for every 10 minutes were created for a total of 195 TS to simulate the whole length of IDM in one day.

Other characteristics of the optimization problem are: the set $a \in A_{p}$ contains 19 bidding areas (the dark areas in Fig 5) for GME or 3 bidding areas (the areas with diagonal lines in Fig 5) for OMIE when simulating only DA for GME or OMIE; 30 bidding areas (no-color areas in Fig 5) when simulating the $\mathrm{CT}$ areas and all the 52 bidding areas when simulating the DA-CT coordination.

The model was tested in three different market operating conditions. In order to validate the numerical performances of the algorithm when executed for real-size problem and investigate the impact to the hybrid IDM mechanism for Option $\mathbf{A}$ and $\mathbf{B}$ :

- Benchmark: The current state-of-the-art at the beginning of 2018 of the European IDM, in which there is no coupling between DA and CT areas. This option was achieved by setting the capacity of interconnectors between DA and CT areas to zero obtaining, thus, independent DA and CT market areas. The results of this case study are used to evaluate the reliability of the data and the numerical performance of the proposed model;

- Option A: Currently, partially implement as OMIE is integrated as of June but GME is not. Here, the coordination of DA-CT is opened for periods following the closure of DA or CRA, all the bids not cleared in the current DA are removed from SOB before starting a new DA but they are allowed to participate in the CTs between the two DAs. This can be achieved through the parameter $T_{s}^{r}$ (only single period orders in DAs). The parameter $T_{s}^{r}$ of bids covering the periods of the next DA auction is set to 1 (which is always smaller than the value of the current $t s$ ), while the $T_{s}^{r}$ of bids covering the periods not to be auctioned is set as the bids in CT, thus allowing these bids to match with bids which come during the DA-CT coordinated period;

- Option B: A possible future scenario for the European IDM. Here, there are only three DAs in GME: MI1, MI2 and MI3 (see Fig. 3a). Thus, in order to use the same data to simulate Option B the following procedure was applied: first, comparing the timescales of Fig. 2a with Fig. 3a it is clear that the MI3 and MI5 of Option A are missing from Option $\mathbf{B}$ while MI1, MI2 and MI4 of Option A are equivalent to MI1, MI2 and MI3 of Option B; thus, all the bids in MI3 and MI5 are converted to bids in the CT with incoming time $\left(T_{s}^{i}\right)$ and removing time $\left(T_{s}^{r}\right)$ defined with respect to the GCT, 240 minutes and 60 minutes before the dispatching period, respectively. This assumption is acceptable since the distance between two auctions is large and the players in GME have a chance to adjust the schedule dispatch by the CT mechanism. Meanwhile, the bids of MI1, MI2, and MI4 of Option A are now the bids of MI1, MI2, and MI3 of Option B. Because the trading of the CT in OMIE is for the whole day, the parameter $T_{s}^{r}$ of all bids covering the periods to be auctioned in the next DA has the same value with the parameter $T_{s}^{i}$ of all bids in the next DA. Meanwhile, the bids in OMIE of the periods not to be auctioned anymore behave as in Option A.

Moreover, it should be also noted that all the market operating conditions use the same set of bids making, thus, the analysis insensitive to bidding behaviour change and allowing the impact of the various market operating conditions be precisely evaluated. The analysis of the bidding behaviour is, in this case, constrained by the absence of information about the generating plants represented in the bids and the companies that controls them. Thus the absence of the cost structure of the power plants makes any assumption in the evolution of the bids arbitrary, so conclusion may be unrealistic.

All scenarios have been solved with a desktop PC equipped with an Intel(R) E5-1620 v3 @ 3.5 GHz processor and 32 Gb RAM. The OM is modeled and solved in GAMS 24.0.2 modeling environment [40], using the CPLEX solver. In order to guarantee that the best possible feasible solution is found by CPLEX, the optimality gaps where set to zero. The DPM is implemented in MATLAB 2015b [41]. The database of all cases study is created in Excel Office [42].

\subsection{Results}

\subsubsection{The Benchmark}

Numerical performance of the algorithm. The numerical performance of the proposed model is here evaluated for the Benchmark. However, similarly results were reported for the other operating conditions. Fig 6 shows the characteristics of the Optimization Problem in the OM for all the sessions in terms of total number of variables, of binary variables and constraints. There are 207 iterations made of: $195 \mathrm{TSs}, 6$ DAs of OMIE, 5 DAs of GME, and 1 DA of Germany. It can be seen that the size of the optimization problem is very large since the maximum number of constraints is 74472 , of variables is 61035 , and of binary variables is 3918 .

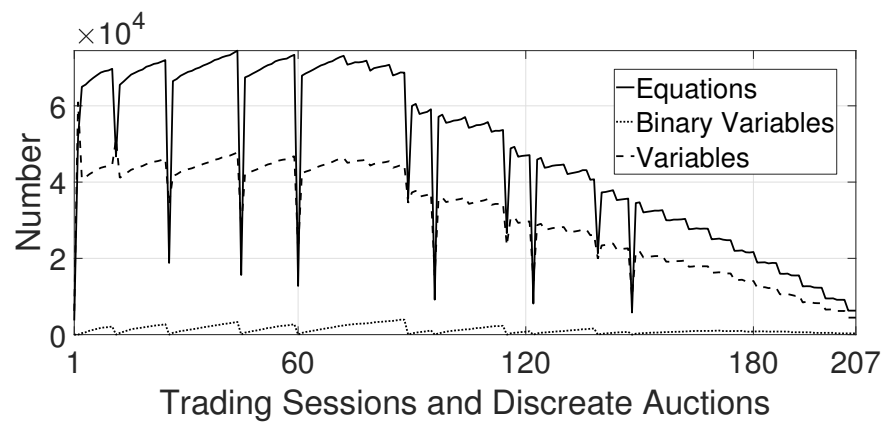

Figure 6: The report of number of: (i) variables, (ii) binary variables, and (iii) constraints for the Benchmark 
Moreover, a progressive reduction of the number of variables and, thus, of constraints, with the advance of the market sessions is noticed in Fig. 6: the cause of this is the progressive reduction of the time validity of the market sessions (see second paragraph of subsection 2.1) with the advance of time, e.g. for the market session at period 1 bids can be submitted for period 1 till the end of the day while for the market session at period 17 bids can be submitted from period 17 till the end of the day. Additionally, the next day IDM $(\mathrm{D}+1)$, for which the bids can be submitted from 14:00 of the current IDM, is not considered in this simulation.

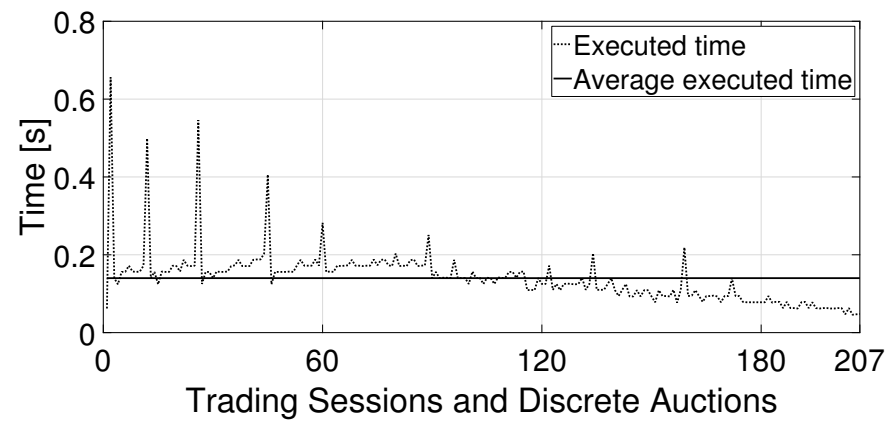

Figure 7: The report of executed time for all sessions of the Benchmark

The computation time of the proposed model is shown in Fig. 7 for the Benchmark. It is clear that the performance of the proposed model is very good since the executed time of one iteration took: maximum $0.656 \mathrm{~s}$, minimum $0.046 \mathrm{~s}$, and in average $0.14 \mathrm{~s}$. These values are well suitable to the requirement of the integrated European IDM where the solution has to be provided immediately for CT and in less than 10 minutes for DA [7].

Analysis of the solution. First of all, the market solutions in DA (GME, OMIE), and CT are compared to the real results in order to validate the data. The comparisons for GME and OMIE revealed that the total accepted volume and marginal price are exactly the public results in [28] and $[29]$ (error $=0 \%$ for all periods).

Table 3: The deviation of the simulated with respect to the real prices in percentage in EPEX Spot

\begin{tabular}{cccc}
\hline Criteria (\%) & France & Germany & Switzerland \\
\hline Max [period] & $36[17 \mathrm{~h}]$ & $22.38[5 \mathrm{~h}]$ & $13.2[13 \mathrm{~h}]$ \\
\hline Min [period] & $1.27[3 \mathrm{~h}]$ & $0.37[13 \mathrm{~h}]$ & $0.23[4 \mathrm{~h}]$ \\
\hline Average & 10.51 & 5.18 & 4.54 \\
\hline
\end{tabular}

For the CT, the EPEX Spot market is analyzed in detail since the same procedure was used to generate the data for all CT markets; the conclusion formulated here also hold for the remaining areas. Here, the maximum price (max_price) of accepted orders in EPEX Spot is presented in Fig. 8 and the deviation in percentage of the

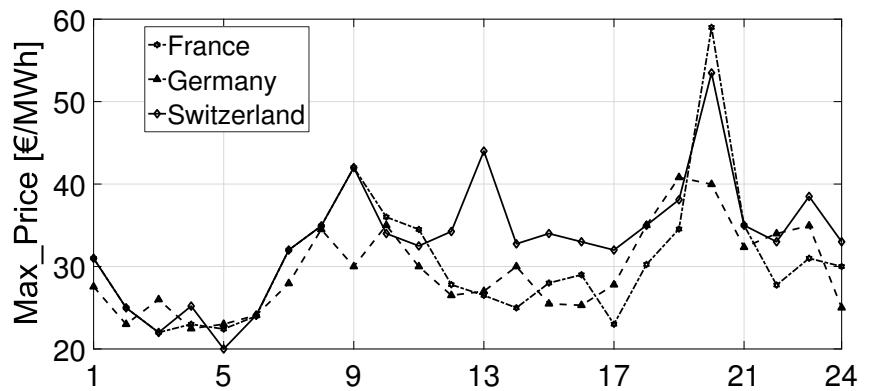

(a)

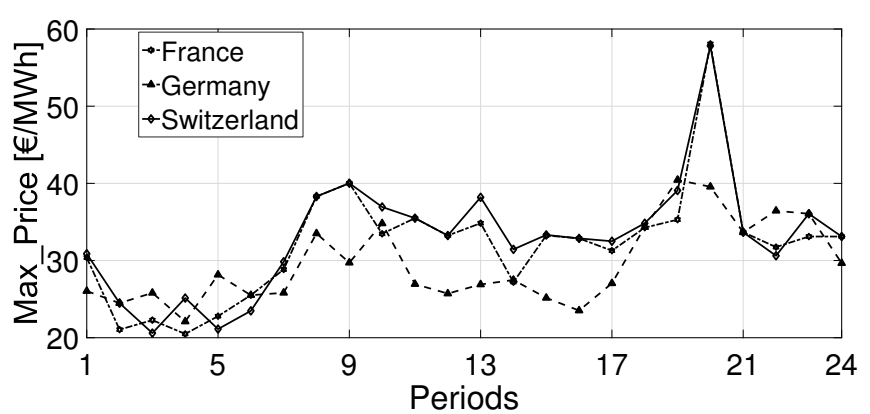

(b)

Figure 8: Maximum prices of IDM in EPEX Spot: (a) the real case and (b) the simulated case

simulated and the real result is shown in Table 3 to confirm the reliability of the data generation procedure. In the real case (see Fig. 8a), the lowest of max_price is between 2 am and 5 am, then it starts increasing; the highest of max_price is between $19 \mathrm{pm}$ and $21 \mathrm{pm}$. This behaviour of the max_price is repeated in the simulated case (see Fig. 8b) Moreover, the average deviation is not high: about 5\% for Germany and Switzerland, and about 10\% for France. These errors occur because in real life, the price of one period in CT changes when a new bid arrives and, thus, since this information is not available it is very difficult to obtain a result very close to the real one with randomly generated data and coming, removing time. Additionally, the maximum deviation of the price (see Table 3) does not happen at the same period in the three countries of EPEX Spot. Therefore, it can be said that the generated data for CT is acceptable.

In order to test the ability of the proposed model to match different product orders (quarterly, half-hourly and hourly orders) and with different incoming times, the hourly orders in GME and OMIE are decomposed into hourly, half-hourly and quarterly orders. The results revealed that the product orders can match each other since the calculated accepted volume and marginal price are the same with the normal condition (all hourly orders).

Finally, the total accepted volume of supply and demand in the entire Europe are shown in Table 4. Here, the second column indicates the amount of volume of SOs that were matched with MSOs (Column 3). The second and third columns of Table 4 show that the market rule of MSOs, in which MSOs can only match with SOs, is ful- 
Table 4: The trading volume of the Benchmark in MWh

\begin{tabular}{ccccc}
\hline & SO $^{\text {ms }}$ & MSO & DA & CT \\
\hline Supply & 159.9 & 783.37 & 198226.91 & 172210.41 \\
\hline Demand & 782.3 & 159.9 & 198226.91 & 171304.44 \\
\hline
\end{tabular}

filled. There is a small difference (1.07 MWh) between the accepted volume of SOs that matched with MSOs from demand side (the second row of column one) and MSOs from supply side (the first row of column two) because there are some SOs from demand side in $22^{\text {th }}$ and $23^{\text {th }}$ periods which are supplied by MSOs through DC interconnectors with non-null losses. The fourth column shows the accepted volume in DA (GME and OMIE), in which the total accepted volume of supply and demand are equal because the interconnectors in GME and OMIE are lossless. Finally, the last column shows the accepted volume in $\mathrm{CT}$; here the total accepted volume of supply is higher than demand because of the losses on the DC interconnectors, e.g. NorNed (NO2-NL), Baltic Cable (DE-SE4), BritNed (GB2-NL), IFA (GB1-FR).

To sum up, the model and the data are reliable for further analysis since all the constraints worked correctly and, considering the missing real data issue, the small average errors between real and calculated results are contained.

\subsubsection{Global analysis: economical metrics}

Next, the operation of the European IDM in the three operating conditions is simulated using the proposed model. First of all, the $\mathrm{TV}^{4}$ and $\mathrm{SW}$ are shown in Table 5. It should first be reminded that in the Benchmark there is no connection between the DA and CT areas as OMIE and GME are not integrated. Option A and Option B represent future possible situations and are simulated by considering the current ATC of interconnectors between the DA and CT areas (the congestion scenario) and by considering a very high value for the ATC of the same interconnectors (the non-congestion scenario). The interconnectors inside the DA and CT areas are kept to the real values in both scenarios.

Table 5: The global criteria for three cases study

\begin{tabular}{|c|c|c|c|c|c|}
\hline \multirow[t]{2}{*}{ Scenario } & \multirow{2}{*}{$\begin{array}{l}\text { Operating } \\
\text { condition }\end{array}$} & \multirow{2}{*}{$\begin{array}{l}\mathrm{SW} \\
{[\mathrm{M} €]}\end{array}$} & \multicolumn{3}{|c|}{ Trading Volume [GWh] } \\
\hline & & & DA & CT & Total \\
\hline & Benchmark & 20.52 & 426.09 & 313.88 & 739.97 \\
\hline \multirow[t]{2}{*}{ Congestion } & Option A & 20.57 & 427.80 & 321.97 & 749.76 \\
\hline & Option B & 20.03 & 380.97 & 364.28 & 745.25 \\
\hline \multirow[t]{2}{*}{ Non-congestion } & Option A & 21.07 & 437.52 & 357.85 & 795.36 \\
\hline & Option B & 20.80 & 390.35 & 439.87 & 830.22 \\
\hline
\end{tabular}

${ }^{4} \mathrm{TV}$ is computed by the sum of the supply accepted volume and the demand accepted volume.
From Table 5, it results that, in general, the total SW and TV of the non-congestion scenarios are higher than that of either the Benchmark or congestion scenarios. It means that the impact of current ATC limits is significant in the integrated European IDM. From the last column of Table 5, it results that the liquidity of the European IDM increases since the TV of all cases are higher than the TV of the Benchmark. However, in the third column of Table 5, a reduction of SW for Option B congestion (- 0.49 M€) with respect to the Benchmark even if the TV increases $(5.28 \mathrm{GWh})$ can be noticed. It is due to the difference in market design between CT, where the market is executed whenever a new bid arrives, and DA, where the market is solved at a fixed point in time: with the same submitted bids but different coming time $\left(T_{s / m}^{i}\right)$, the SW of CT can be smaller than that of DA (as explained in the simple example of Appendix A).

Therefore, the focus is put on investigating in details the total TV of DA (in the fourth column) and CT (in the fifth column):

- DA: In Option A, because of the two CRAs in GME, the TV of DA increased in comparison to the Benchmark with 1.71 GWh for the congestion scenario and with $11.43 \mathrm{GWh}$ for the noncongestion scenario. Meanwhile, in the Option B, the TV of DA in both cases decreased in comparison to the Benchmark because of the lack of two DAs (MI3 and MI5 in Option A) in GME, e.g. - 45.12 GWh for the congestion scenario and - 35.74 GWh for the non-congestion scenario;

- CT: In general, because of the participation of GME and OMIE in the CT, the TV in CT increased in all cases with respect to the Benchmark. The TV in CT of Option B is always higher than that of Option A because Option B has a larger degree of DA-CT coordination.

Table 6: The revenue of supply and the expenditure of demand for PXs of the Benchmark in M€

\begin{tabular}{ccc}
\hline PXs & Supply & Demand \\
\hline GME & 4.784 & 4.795 \\
\hline OMIE & 1.989 & 1.99 \\
\hline REST & 3.409 & 4.637 \\
\hline
\end{tabular}

Table 6 presents the revenue of supply and the expenditure of demand for the Benchmark of GME, OMIE, and the REST of PXs (Nordpool Spot, APX, EPEX Spot and Belbex) in the last row. They were calculating according to the payment schemes of the market mechanism: payas-bid for CT and marginal zonal price for DA. Moreover, Fig. 9a and Fig. 9b show the deviation of the revenue of supply and the expenditure of demand of the congestion and non-congestion scenarios with respect to the 


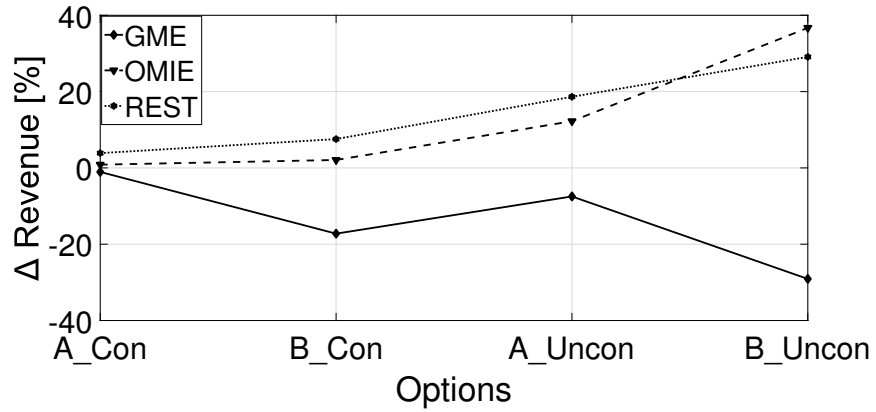

(a)

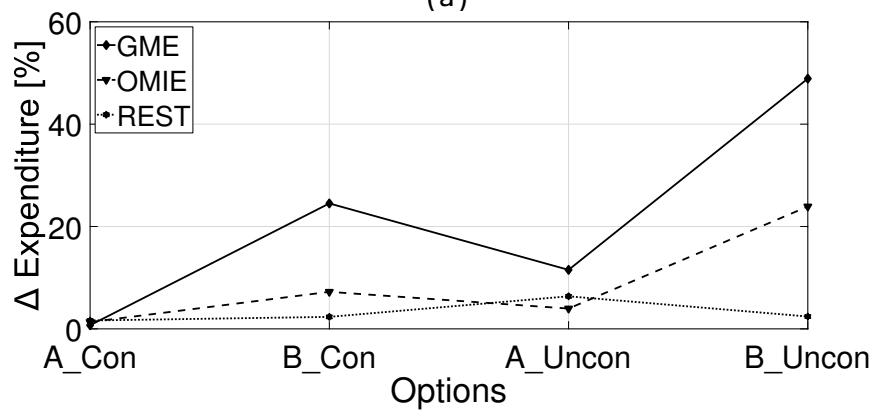

(b)

Figure 9: The deviation of the congestion and non-congestion scenarios respect to the Benchmark for: (9a) Supply revenue, and (9b) Demand expenditure

Benchmark, respectively. In Fig. 9a and Fig. 9b, the supply revenue and the demand expenditure of OMIE and the REST of PXs increase in comparison to the Benchmark. Therefore, the integrated European IDM brings a positive signal to both supply and demand in OMIE and the REST of PXs. Meanwhile, the revenue of supply decreases in Fig. 9a and the expenditure of demand increases in Fig. 9b for GME. It means that the TV of the supply of GME decreases and the TV of the demand of GME increases in the integrated European IDM. This is due to the two CRAs in GME, in which the demand has a trend to buy energy from outside of GME where the supply bids are cheaper, thus the suppliers in GME become uncompetitive. By observation of Fig. 9, it results that Option $\mathbf{B}$ has a stronger impact on the market result than Option A because the apexes of Option $\mathbf{B}$ are always higher than those of Option $\mathbf{A}$ for the positive values and viceversa for the negative values (the supply of GME in Fig. 9a) in both congestion and non-congestion scenarios when compared independently.

\subsubsection{Global analysis: impact of ATC limits}

Fig. 10 shows the imported and exported energy of GME and OMIE for the congestion and non-congestion scenarios. Here, the negative values stand for importing energy and the positive values for exporting energy. The continuous line is representative for GME, while the dashed line for OMIE. Fig. 10 confirms that the current ATC of the interconnectors of both OMIE-EPEX Spot and GME-EPEX Spot are limiting the transfer energy in the integrated IDM structure: it varies between -4317 MWh to 4447 MWh for the non-congestion scenario and between

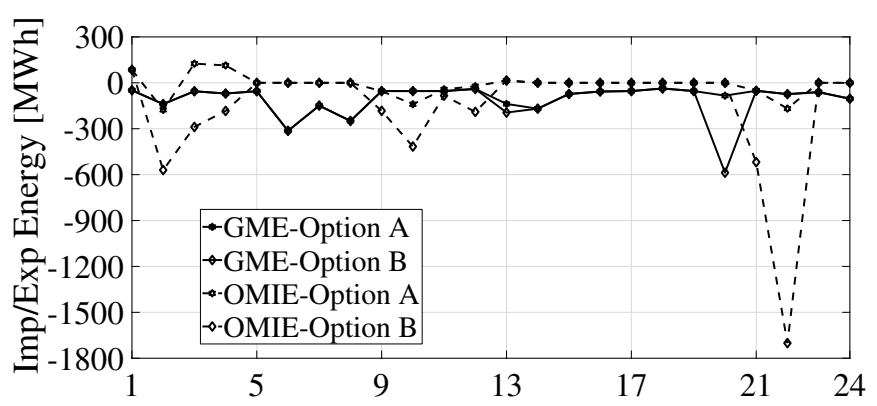

(a)

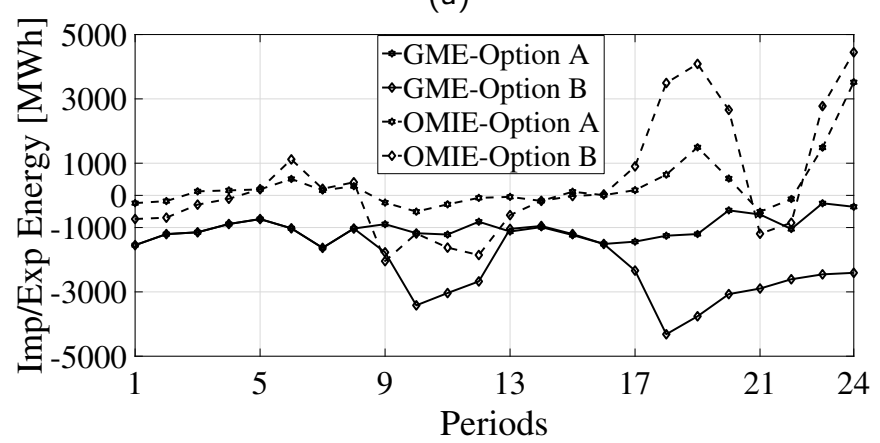

(b)

Figure 10: The import and export energy of GME and OMIE in one day: (10a) Congestion and (10b) Non-Congestion

-1701 MWh to 125 MWh for the congestion scenario. Fig. 10 also shows that GME tends to buy energy from other countries, since the lines are always below zero for all periods in both scenarios. Meanwhile, OMIE can either import or export energy but the current ATC limits reduce the chance for players in OMIE to export energy: the amount of exported energy are significantly reduced in the congestion scenario (see Fig. 10a) with respect to the non-congestion scenario (see Fig. 10b), e.g. in Option A 339.2 MWh are exported in the congestion scenario and 9366.76 MWh in the non-congestion scenario; in Option B $0 \mathrm{MWh}$ is exported in the congestion scenario and 20314.8 MWh in the non-congestion scenario.

To better understand the restrictions of the actual ATC limits, Fig. 11 represents the ones initially available to the IDM market. It is reminded that the ATC limits of the IDM strongly depend on the margins remaining after the execution of the DAM and, thus, they can have very stringent values. Fig. 11a shows that, with the exception of the first four periods, OMIE cannot export energy, which confirms the results of Fig. 10. Fig. 11b shows only the ATC limits in importing around GME area since GME tends to import and the export limits are very high. It can be noticed that among the three intra-border interconnectors (FRAN-FR, SVIZ-CH, and AUST-DE), except period 20, only the SVIZ-CH one has available margin. However, the flow on this interconnector is determined by the flows on CH-FR and CH-DE, among which only $\mathrm{CH}-$ FR has available capacity. It results that GME can only import through the radial path SVIZ-CH and $\mathrm{CH}-\mathrm{FR}$, so the actual available capacity is given by the most limiting between the two. 


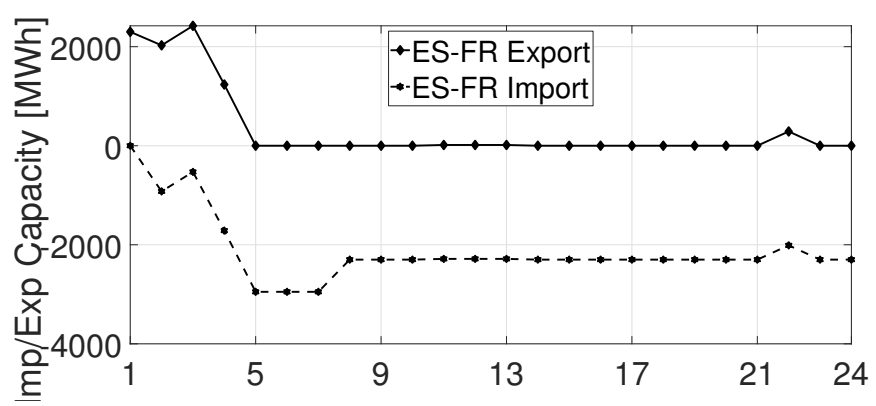

(a)

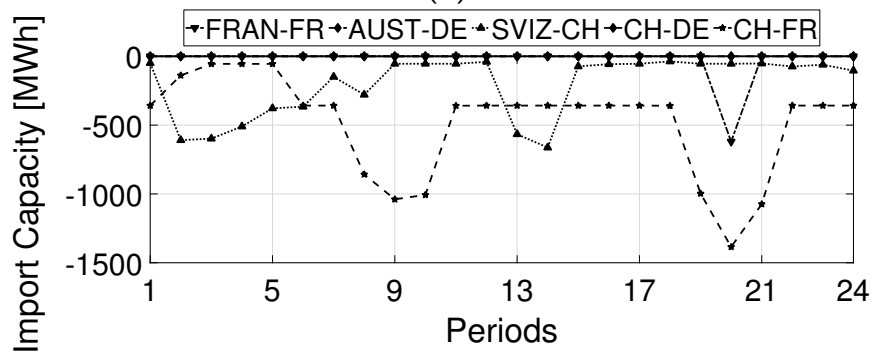

(b)

Figure 11: Available capacity before of the IDM: (11a) OMIE border and (11b) GME border

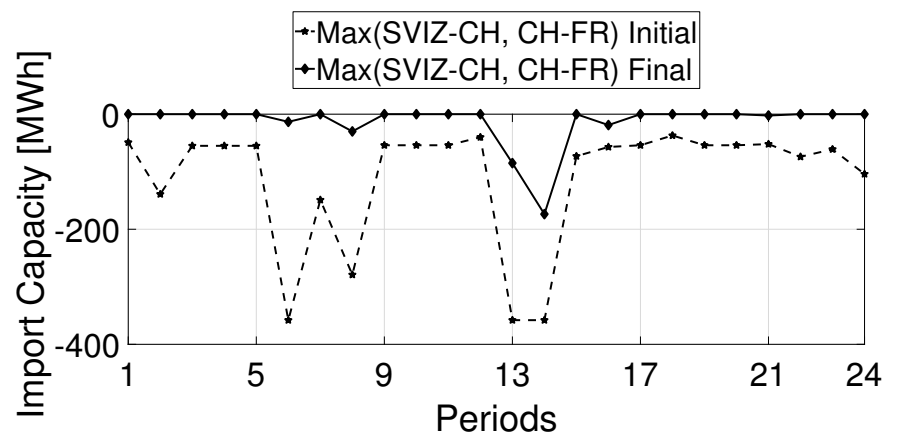

Figure 12: Available capacity on the radial path SVIZ-CH and CHFR before and after the IDM

Fig. 12 shows the most limiting of the two (the maximum, as they are negative numbers) before and after the execution of the IDM, only for Option A-congestion case since the energy exchange of Option B-congestion is almost the same (see Fig. 10). It can be observed that the available import capacity is very limited and that in almost all the hours it is fully exploited by the IDM.

Period 21 represents a particular case for GME. Here, only the FRAN-FR intra-border interconnector allows GME import. When compared against Fig. 10b, Fig. 11b shows that the available capacity is not fully exploited in both Options.

In general, the presence of congestions gives rise to congestion costs, also known as the dead-weight loss, as the optimal economical surplus of the market (the SW) is negatively affected. The congestion cost can be calculated as the SW difference between the non-congestion and congestion scenarios for the two Options, separately. With reference to Table 5, globally, Option B produces higher congestion costs: Option A registers a loss in the SW of
Table 7: The SW congestion and the congestion cost of both Options in $\mathrm{M} €$

\begin{tabular}{cccc}
\hline Options & PXs & SW-congestion & Congestion cost \\
\hline \multirow{3}{*}{ A } & GME & 12.11 & -0.86 \\
\cline { 2 - 4 } & OMIE & 6.94 & 0.17 \\
\cline { 2 - 4 } & REST & 1.52 & 0.19 \\
\hline \multirow{3}{*}{ B } & GME & 11.57 & -1.77 \\
\cline { 2 - 4 } & OMIE & 7.03 & 0.36 \\
\cline { 2 - 4 } & REST & 1.43 & 0.64 \\
\hline
\end{tabular}

about $2.8 \%$ while Option B a loss of about $3.7 \%$. In details, Table 7 shows the distribution of the congestion costs among the three analyzed macro-areas: GME, OMIE and the CT areas (REST in Table 7). The importing area, i.e. the GME, is registering a significant cost, while the exporting areas are registering gains, especially the $\mathrm{CT}$ areas.

\subsubsection{The impact of the two options on all TSs in CT}

Firstly, the TV of all TSs is shown in Fig. 13 for the Benchmark. Here, the maximum TV is $4093 \mathrm{MWh}$ at $7^{t h}$ TS and the minimum TV is zero. It should be emphasized that the TV decreases with time because the trading periods get shorter, result which is coralated with the optimization problem characteristics of Fig. 6.

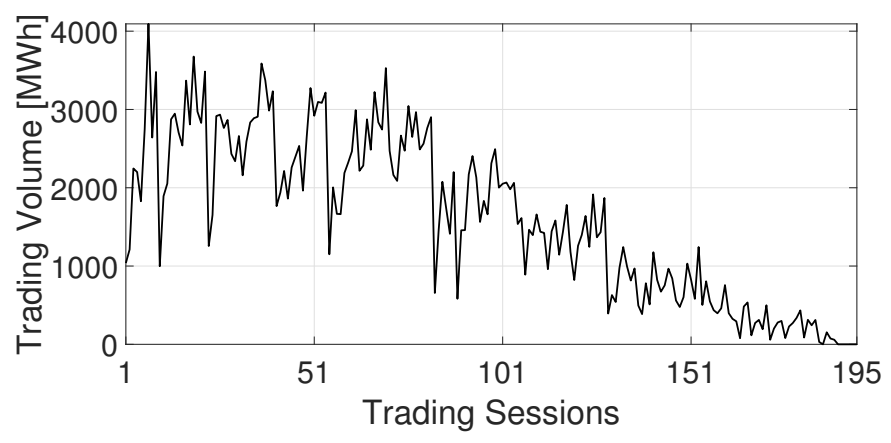

Figure 13: The TV of all TSs in the CT of the Benchmark

Fig. 14 shows the deviation of both Option $\mathbf{A}$ and Option B with respect to the Benchmark for the congestion scenario (Fig. 14a) and the non-congestion scenario (Fig. 14b). The results indicate that the TV in CT of Option B is higher than that of Option A for both scenarios, considered separately. Moreover, these finding also show that the TV in CT of the non-congestion scenario is more than that of the congestion scenario, e.g. the maximum deviation of $\mathrm{TV}$ of the congestion scenario is $4054 \mathrm{MWh}$ in the $171^{\text {th }} \mathrm{TS}$, meanwhile in noncongestion scenario, the deviation of TV in the $171^{\text {th }} \mathrm{TS}$ is $5271 \mathrm{MWh}$ and the maximum is $7083 \mathrm{MWh}$ in the $147^{\text {th }}$ TS.

Moreover, the TV of GME and OMIE for all TSs in the CT are also shown in Table 8 for the total TV, and Fig. 15 and Fig. 16 for each TS, respectively. The TV 


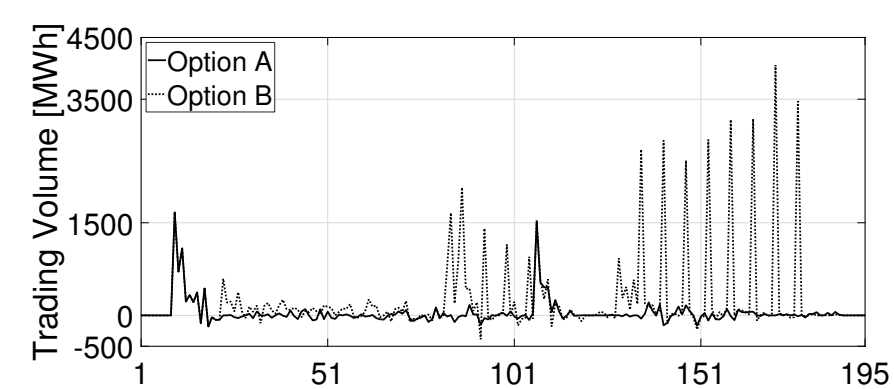

(a)

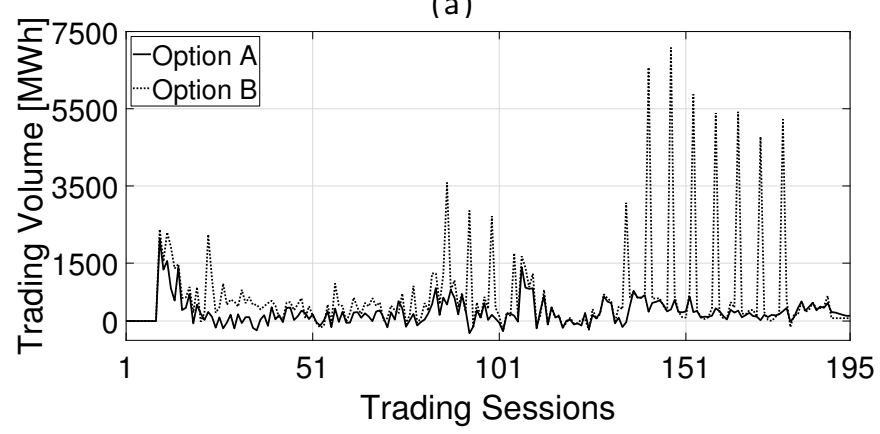

(b)

Figure 14: The deviation of TV of Option A and Option B with respect to the Benchmark: (14a) Congestion and (14b) NonCongestion

Table 8: The total TV of GME and OMIE in all TSs in the CT in MWh

\begin{tabular}{cccc}
\hline Cases & Options & GME & OMIE \\
\hline Congestion & A & 525 & 1736 \\
\cline { 2 - 4 } & B & 30742 & 7462 \\
\hline Non-congestion & A & 13395 & 13401 \\
\cline { 2 - 4 } & B & 55305 & 46439 \\
\hline
\end{tabular}

of GME and OMIE in the CT of Option B are always higher than that of Option $\mathbf{A}$ in both congestion and non-congestion scenarios when compared separately for each of the two scenarios (see Table 8 and the curves representing Option B are always above the curves representing Option A in Fig. 15 and Fig. 16). This can be seen when comparing first row against second row of Table 8 for the congestion scenario, third row against forth row of Table 8 for the non-congestion scenario, from Fig 15a and Fig. 16a for the congestion scenario, and Fig. 15b and Fig. 16b for the non-congestion scenario. In general, these results show that a higher degree of DA-CT integration (Option B) leads to an increase in the TV with respect to a lower degree of coordination (Option A). When the impact of the ATC limits on each Option is evaluated (first row against third row and second row against forth row of Table 8, respectively) a large increase of the TV for both Options is noticed. This result confirm that the current ATC limits are very stringent.

It is interesting to note in Table 8 that the TV for GME in Option B congestion scenario (30742 MWh) is higher than that in Option A non-congestion scenario

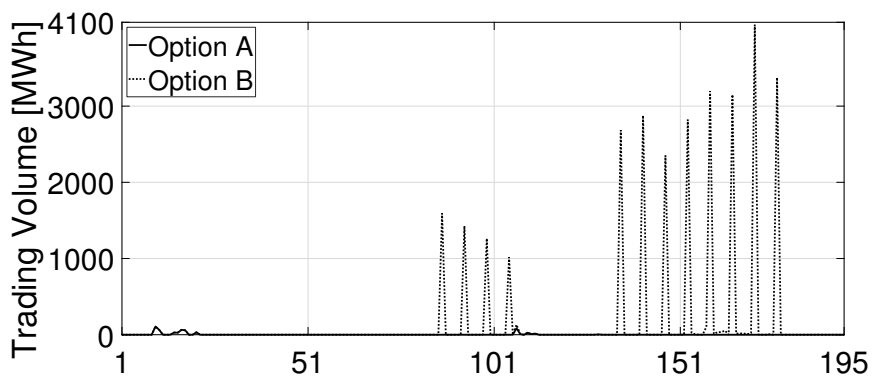

(a)

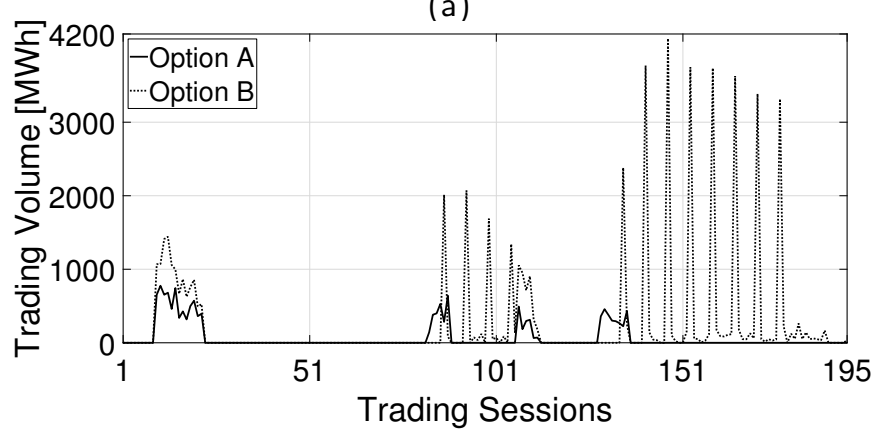

(b)

Figure 15: The TV of all TSs in CT of GME: (15a) Congestion and (15b) Non-Congestion

(13395 MWh). This is explained by the lack of the two DAs of Option A in Option B and their insertion in the CT of Option B, where they are cleared mostly locally due to the presence of the ATC limits. In details, Option B imports a high TV from the DAs of Option A, this TV does not appear in the numbers reported in Table 8 for Option A but it appear in Option B.

Fig. 15 shows that for Option $\mathbf{B}$ the TV of GME in the CT significantly increases in some TSs in the congestion and non-congestion scenarios, i.e. from $87^{\text {th }}$ TS to $105^{\text {th }}$ TS and from $135^{\text {th }}$ TS to $177^{\text {th }}$ TS, while the maximum TV over all TSs of the congestion scenario (4067 MWh) is almost the same with that of the noncongestion scenario (4137 MWh). This is again explained by the lack of the two DAs of Option A in Option B and their insertion in the CT of Option B, where they are cleared. Moreover, this is what gives the spikes of Option B in Fig. 14 and Fig. 15, respectively.

Regarding Fig. 16, it is interesting to point out that the shape of either Fig. 16a and Fig. 16b is more or less the same between the $1^{\text {st }}$ TS and the $140^{\text {th }}$ TS but not for the rest, in particular for Option B of the noncongestion scenario. In this case, the suppliers in OMIE can match with demand orders from the entire Europe, including GME, as there is ATC available. In fact, for the TSs for which the spikes in Fig. 16b are recorded the exports from OMIE increase and, at the same time, a similar amount of energy is imported by GME; e.g. in the $141^{\text {th }}$ TS, OMIE exports 2934 MWh while GME imports 2560 MWh. Thus, it can be said that the cheaper generators from OMIE push the more expensive generators of GME 


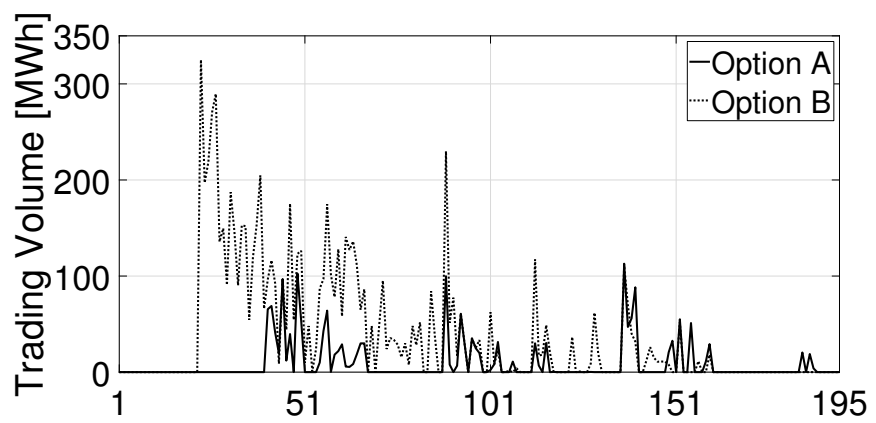

(a)

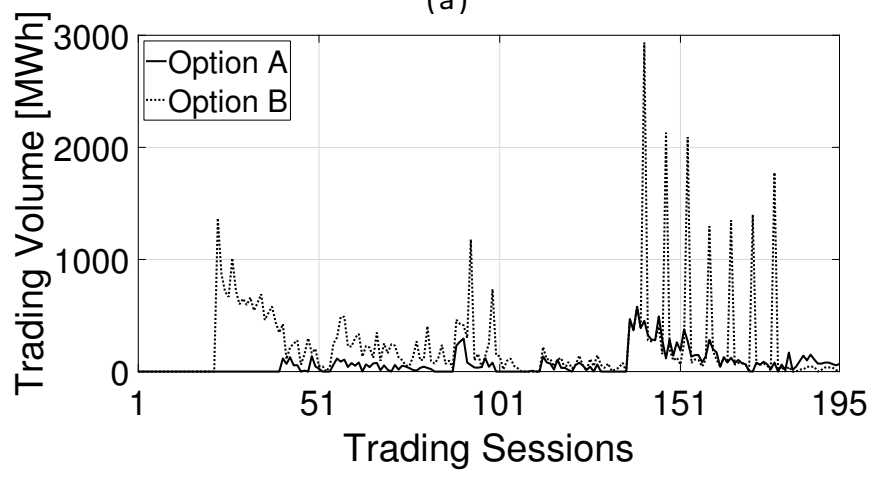

(b)

Figure 16: The TV of all TSs in CT of OMIE: (16a) Congestion and (16b) Non-Congestion

in the out-of-price area: the demand bids of the GME are matched against those from other countries while the surplus of generation in OMIE is exported. This happens in Option B for the CT TSs where the bids of the missing GME DAs of Option A are present (compare Fig. 16b with Fig. 15b).

\subsubsection{The impact of the two options on DAs in GME and OMIE}

First of all, the SW in M€, TV in MWh, and Volume Weighted Average Price (VWAP) in $€ / \mathrm{MWh}^{5}$ of each DA of the Benchmark are presented in Table 9. Then the deviation of SW in $€$, TV in MWh, and VWAP in percentage of Option A and Option B with respect to the Benchmark are shown in Table 10 for the congestion and non-congestion scenarios. Here, VWAP is defined as the volume weighted average price to buy or sell $1 \mathrm{MWh}$ using $(34)^{6}$. In these Tables, the DAs of GME are denoted by MI1-MI5 and the DAs of OMIE are denoted by SO1SO6. Finally, as explained in Section 5.1 it is reminded that the MI3 of Option B corresponds to MI4 of Option A reason why the results of Option B are given for MI4 instead of MI3.

${ }^{5}$ The payment method in DA based on the market clearing price.

$$
V W A P=\frac{\sum_{a \in A_{p}} \sum_{t \in T_{p}^{h}} \sum_{s \in S_{a}}\left(x_{s}^{t} \cdot Q_{s}^{t} \cdot \lambda_{a}^{t}\right)}{\sum_{a \in A_{p}} \sum_{t \in T_{p}^{h}} \sum_{s \in S_{a}}\left(x_{s}^{t} \cdot Q_{s}^{t}\right)}
$$

where $\lambda_{a}^{t}$ is the Lagrange multiplier of the balancing equation (5), which is also equal to the market clearing price of area $a$. 
As explained in Section 4.1, only two DAs are operated as CRA, i.e. MI2 and MI4 of GME while the others (including OMIE DAs) are operated in an uncoupled fashion. For this reason, the impact of the two Options on the DAs sessions is major for only the MI2 and MI4 (two grey columns of Table 10). Here, the effect is very positive in both congestion and non-congestion scenarios when compared to the Benchmark: (i) the SW increases by up to $22096 €$ in the congestion scenario (MI2, both Options) and up to $72192 €$ in the non-congestion scenario (MI4, Option A); (ii) the TV increases by up to 1518 MWh in the congestion scenario (MI2, both Options) and up to $5813 \mathrm{MWh}$ in the non-congestion scenario (MI2, both Options); (iii) the VWAP decreases by up to $-1.43 \%$ in the congestion scenario (MI2, both Options) and up to $-18.88 \%$ in the non-congestion scenario (MI4, Option A). Clearly, the results of the non-congestion scenario are significantly better than the results of the congestion scenario for both Options, which shows the limiting impact of the tight ATCs on the DA-CT interconnectors. Finally, it is interesting to note that the results for MI2 in both Options are identical for both scenarios: this is because up to the MI3 session, the two Options are identical (MI3 is in Option A but is missing from Option B).

For the sessions which are not operated as CRAs (white colors of Table 10), so are still uncoupled, the impact of market integration is minimal and indirect: the changes that can be seen in Table 10 are very small when compared to the results of MI2 and MI4 and they are due to the update of the ATC of the interconnectors inside the DA areas during the previous CT and/or CRA sessions. The scale of these updates are small as the interconnectors inside the DA areas are already congested (or close to congestion) in the DAM and the IDM solution is pushing the flows in the congested direction in most of the cases. However, these small changes are generally positive since the SW and the TV register small increases with respect to the Benchmark while the VWAP generally registers small decreases with respect to the Benchmark.

The impact of the two CRAs on the TV of participants in GME is investigated separately for the supply and the demand side in Table 11. Here, the deviations introduced by the two Options with respect to the Benchmark is shown. The TV of supply and demand in the Benchmark is the same because the interconnectors inside GME do not have losses in the market model, i.e. $24533 \mathrm{MWh}$ for MI2 and 12696 MWh for MI4. Finally, it should be noted that the results in Table 11 only represent the supply and demand inside GME, while the results of CRAs in Table 10 consider the entire study case.

Qualitatively, the results of Table 11 are: (i) the TV of supply always decreases while the TV of demand always increases; (ii) in absolute values the TV of the demand is always smaller than the TV of the supply and (iii) in absolute values, the results of the non-congestion scenario are much higher than the results of the congestion scenario. 
Table 11: The TV of supply and demand of CRAs of Option A and Option B in GME in MWh

\begin{tabular}{cccccc}
\hline \multirow{2}{*}{ Options } & \multirow{2}{*}{ DAs } & \multicolumn{2}{c}{ Congestion } & \multicolumn{2}{c}{ Non-congestion } \\
\cline { 3 - 6 } & & Supply & Demand & Supply & Demand \\
\hline \multirow{2}{*}{ A } & MI2 & -1012 & 535 & -3598 & 2715 \\
\cline { 2 - 6 } & MI4 & -185 & 0 & -2324 & 2739 \\
\hline \multirow{2}{*}{ B } & MI2 & -1012 & 535 & -3598 & 2715 \\
\cline { 2 - 6 } & MI4 & -246 & -5 & -2001 & 2398 \\
\hline
\end{tabular}

These results show that, on one hand, the more expensive generators of GME loose the opportunity to trade in front of the cheaper generation available in the areas outside the GME. Moreover, this also determines the increased of the accepted demand from inside the GME. Finally, the neat difference between the congestion and non-congestion scenarios shows, again, the limiting impact on the TV of the currently tight ATCs on the DA-CT interconnectors.

\section{Conclusion}

According to the requirements of the Continuous Trading Matching Algorithm, the paper developed a unified mathematical model to run the integrated European IDM. Moreover, an iterative process is proposed to run a whole length of IDM in Europe with any level of coordination between DA and CT. The numerical performance of the model and of the iterative process is validated by a realistic case study which is created based on the current European IDM structure and data. Two official DA-CT coordination proposals, one of GME and one of OMIE are simulated by the iterative process. These proposals show many advantages of the single European IDM, however, there are some disadvantages as well.

In detail, the integrated European IDM seems very promising since the players of both GME and OMIE can approach the cheaper bids from other countries. Therefore, this implementation also requires Italian and Iberian suppliers to change their behaviour and bid in a profitable way. However, the more expensive suppliers, e.g. those of GME, become uncompetitive and they would need to change their bidding strategy or invest to make the plants more cost-efficient or even invest into cost-efficient plants. To clearly understand the optimal behaviour, other types of mathematical approaches are required, e.g. game theory or reinforcement learning. However, their efficient use requires a correct representation of the market rules. The paper provides this under the form of a complex Mixed Integer Linear Problem model, so the building block for future studies has been put.

Moreover, the outcome of the non-congestion scenario is always better than that of the congestion scenario in terms of SW, liquidity and VWAP when they are compared for each Option, separately. Therefore, the insufficiency of the ATC of interconnectors significantly minimizes the positive impact of the integrated European
IDM. The paper went deeper and identified the interconnectors that limit the commercial exchanges between countries and evaluated their financial impact. From this point on, the proposed model can be used to evaluate the changes needed to alleviate the problems: further increase of the transmission capacities and the redefinition of the market areas being among possible investigations that can be performed by the TSOs to define their future strategies.

Finally, the proposed model is also validated by the current evolution of the European IDM. The paper shows that Option B is the better option for the integration of OMIE and GME; however Option $\mathbf{A}$ is more near to the current national market structure, so it is the to go option for a fast integration. In real-life, OMIE has recently been integrated with Option A but is expected to change to Option B. Thus, the proposed model shows the ability to correctly evaluate a real-life situation so it represents a reliable tool for future studies like the ones mentioned in the previous paragraphs and/or others, e.g. the future evolution of the generation park towards greener technologies or future evolution of the market rules. Last but not least, this also means that the PXs can use the proposed model to clear the market.

\section{References}

[1] T. E. parliament, the council of the European Union, Regulation (EC) No 715/2009 of the European Parliament and of the Council of 13 July 2009 on conditions for access to the natural gas transmission networks and repealing Regulation (EC) No 1775/2005 (Text with EEA relevance), http://eur-lex.europa.eu/legal-content/EN/ALL/ ?uri=0J :L:2009:211:TOC (2009).

[2] EPEXSPOT, pcr: Price coupling, https://www.epexspot.com/ en/market-coupling/pcr.

[3] L. H. Lam, V. Ilea, C. Bovo, Impact of the price coupling of regions project on the day-ahead electricity market in italy, in: 2017 IEEE Manchester PowerTech, 2017, pp. 1-6. doi:10.1109/ PTC. 2017.7981215.

[4] E. Commission, Commission Regulation (EU) 2015/1222 of 24 July 2015 (2015).

URL http://eur-lex.europa.eu/legal-content/en/ALL/ ?uri=CELEX : 32015 R1222\&qid $=1496153489245$

[5] TenneT, European target model, day-ahead market coupling. URL https://www.tennet.eu/electricity-market/ german-market/congestion-management/ european-target-model/

[6] E. Commission, XBID Market Project (2015). URL http://www.mercatoelettrico.org/En/Mercati/ MercatoElettrico/XBID.aspx

[7] C. Regulation, Nemos-proposal (2017). URL http://www.europex.org/external-consultations/ all-nemos-consultation/

[8] N. Committee, Intraday Algorithm Requirements (2016). URL http://www.mercatoelettrico.org/En/Mercati/ MercatoElettrico/XBID.aspx

[9] GME, Consultation Paper on Intraday Coupling model for Italian Borders (2017).

URL http://www.admie.gr/fileadmin/groups/EDLES_DHEP/ 2016_Auction_Rules/Consultation_paper_on_complementary_ auctions_final.pdf

[10] OMIE, Operating proposal for the intra-day market in MIBEL (2017)

URL http://www.omie.es/files/OPERATING\%2OPROPOSAL\% 20FOR $\% 20$ THE $\% 20$ INTRADAY $\% 20$ MARKET $\% 20$ IN $\% 2$ MIBEL . pdf 
11] OMIE, New Continuous Intraday Market in MIBEL (2018). URL http://www.nuevomercadointradiario.omie.es/docs/ 20180606_WEBINAR_ENG.PDF

[12] R. Scharff, M. Amelin, Trading behaviour on the continuous intraday market elbas, Energy Policy 88 (2016) 544 - 557. doi:https://doi.org/10.1016/j.enpol.2015.10.045.

URL http://www.sciencedirect.com/science/article/pii/ S0301421515301713

[13] J. Chaves-Ávila, C. Fernandes, The spanish intraday market design: A successful solution to balance renewable generation?, Renewable Energy 74 (2015) 422 - 432. doi:https: //doi.org/10.1016/j.renene.2014.08.017.

URL http://www.sciencedirect.com/science/article/pii/ S0960148114004765

[14] S. Hagemann, C. Weber, Trading volumes in intraday markets: Theoretical reference model and empirical observations in selected european markets, Tech. rep., EWL Working Paper (2015).

[15] K. Neuhoff, N. Ritter, A. Salah-Abou-El-Enien, P. Vassilopoulos, Intraday markets for power: Discretizing the continuous trading?, DIW Discussion Papers 1544, Berlin (2016).

[16] R. Kiesel, F. Paraschiv, Econometric analysis of 15-minute intraday electricity prices, Energy Economics 64 (2017) 77 - 90 . doi:http://dx.doi.org/10.1016/j.eneco.2017.03.002.

[17] C. Pape, The impact of intraday markets on the market value of flexibility - decomposing effects on profile and the imbalance costs, Energy Economics 76 (2018) 186-201.

[18] D. Newbery, G. Strbac, I. Viehoff, The benefits of integrating european electricity markets, Energy Policy 94 (2016) $253-263$. doi:http://dx.doi.org/10.1016/j.enpol.2016.03.047.

URL http://www.sciencedirect.com/science/article/pii/ S0301421516301513

[19] D. Newbery, M. G. Pollitt, R. A. Ritz, W. Strielkowski, Market design for a high-renewables european electricity system, Renewable and Sustainable Energy Reviews 91 (2018) 695-707.

[20] A. Marien, P. Luickx, A. Tirez, A continuous intraday trading model in a cacm fg compliant world, in: 11th International Conference on the European Energy Market (EEM14), 2014, pp. 1-6. doi : 10.1109/EEM. 2014.6861242.

[21] L. H. Lam, V. Ilea, C. Bovo, European day-ahead electricity market coupling: Discussion, modeling, and case study, Electric Power Systems Research 155 (Supplement C) (2018) 80 92. doi:https://doi.org/10.1016/j.epsr.2017.10.003. URL http://www.sciencedirect.com/science/article/pii/ S037877961730398X

[22] L. H. Lam, V. Ilea, C. Bovo, A thorough comparison among various mathematical approaches to compute pun in italy, in: 2018 15th International Conference on the European Energy Market (EEM), IEEE, 2018, pp. 1-5.

[23] I. Savelli, B. Cornélusse, A. Giannitrapani, S. Paoletti, A. Vicino, A new approach to electricity market clearing with uniform purchase price and curtailable block orders, Applied Energy 226 (2018) 618-630.

[24] P. project, Pcr stakeholder (2016). URL http://www.mercatoelettrico.org/En/MenuBiblioteca/ Documenti/20160120EuphemiaStakeholderForum11Jan2016.pdf

[25] P. C. of Regions, EUPHEMIA Public Description (2016). URL https://www.epexspot.com/en/market-coupling/pcr

[26] EPEXSPOT, intra-day lead time, https://www.epexspot.com/ en/product-info/intradaycontinuous/intraday_lead_time.

[27] Noor Pool, intra-day in germany, http://nordpoolspot.com/ TAS/intraday-trading/continuous-intraday-in-germany/.

[28] GME, public-domain bids/offers, http://www . mercatoelettrico.org/En/Esiti/MGP/EsitiMGP.aspx.

[29] OMIE, intraday market, http://www.omie.es/files/flash/ ResultadosMercado.swf.

[30] EPEXSPOT, market data, https://www.epexspot.com/en/ market-data.

[31] EPEXSPOT, epex spot market rule, https://www.epexspot. com/en/extras/download-center.

[32] CWE, The Central West Europe intraday ATC Capacity
Calculation process after Flow-Based Market Coupling (2015). URL http://www.elia.be/en/products-and-services/ cross-border-mechanisms/transmission-capacity-at-borders/ calculation-methods\#anchor6

[33] ENTSO-E, Cross Border Intraday (XBID) Trading Solution Pre-launch Event (2018).

URL http://www.mercatoelettrico.org/En/MenuBiblioteca/ Documenti/20180131_XBIDpresentation_golive_launch_ event.pdf

[34] D. I. Chatzigiannis, G. A. Dourbois, P. N. Biskas, A. G. Bakirtzis, European day-ahead electricity market clearing model, Electric Power Systems Research 140 (2016) 225 - 239. doi:https://doi.org/10.1016/j.epsr.2016.06.019.

URL http://www.sciencedirect.com/science/article/pii/ S0378779616302279

[35] CWE-TSOs, available transfer capacity, http://www. intraday-capacity.com/portal/php/main.php.

[36] Nordpool Spot, elbas initial capacity, http://www . nordpoolspot.com/Market-data1/Elbas/Market-data13/ Initial-capacity/Norway4/?view=table.

[37] Joint Allocation Office, intrday auction, http://www.jao.eu/ marketdata/intradayauctions.

[38] N. Pool, Trading capacities, http://www.nordpoolspot.com/ TAS/Day-ahead-market-Elspot/Trading-capacities/.

[39] NORDPOOL, nord pool intraday statistics, https: //www.nordpoolgroup.com/Market-data1/Intraday/ Market-data1/Market-data1/Overview/?view=table.

[40] GAMS, general algebraic modeling system, https://www.gams . com/.

[41] Mathworks, programming fundamentals, http://www. mathworks.com.

[42] Microsoft, office 365, https://www.microsoftstore. com/store/mseea/it_IT/cat/categoryID.70174300?icid= HP-Spotlight4-Office-051717-it_IT.

\section{A. An example of the execution of Discrete Auc- tion and Continuous Trading}

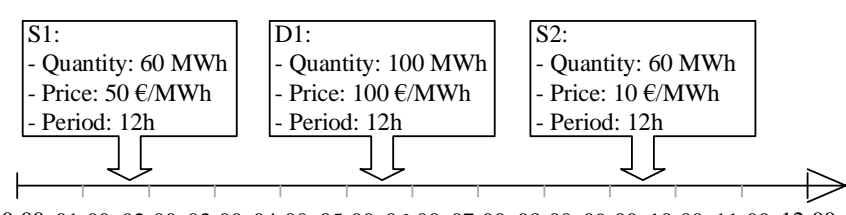

00:00 01:00 02:00 03:00 04:00 05:00 06:00 07:00 08:00 09:00 10:00 11:00 12:00

(a)

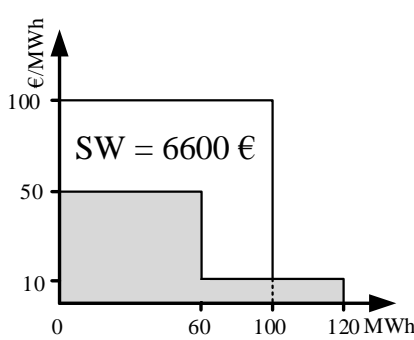

(b)

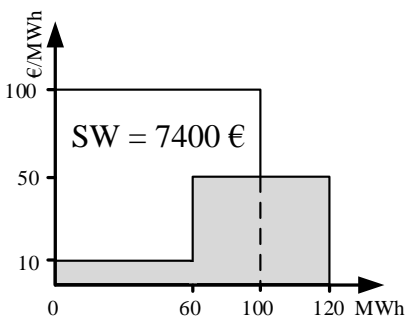

(c)
Figure 17: The example of the execution of DA and CT: (17a) The time-line of submitting bids, (17b) Execution of CT, and (17c) Execution of DA

A simple example is given in Fig. 17a where three bids are submitted to the market at different coming time $\left(T_{s}^{i}\right)$ for the $12^{\text {th }}$ period, including two supply bids (S1 and S2) 
and one demand bid (D1). In CT, the market is executed whenever a new bid arrives, thus the market is executed three times as follows:

1. Up to period 05:00, there is no bid accepted as there is only $\mathrm{S} 1$ in SOB;

2. At period 06:00, $\mathrm{S} 1$ is fully accepted (60 MWh), and D1 is partially accepted $(60 \mathrm{MWh})$. At this point, the SW equals to $3000 €$;

3. At period 10:00, the rest of D1 is fully accepted (40 $\mathrm{MWh}$ ) and S2 is partially accepted (40 MWh). For this CT session, the SW equals to $3600 €$.

Therefore, the total SW is $6600 €$ and the total TV is $200 \mathrm{MWh}$ as shown in Fig. 17b where the equivalent cumulated curved for period 12:00 overall $\mathrm{CT}$ sessions is shown.

If the same bids are run in a DA session for period 12:00 with all the bids, the market is executed at a fixed point in time, it means that all bids are executed at the same time. Therefore, supposing that the market is executed after S2 arrived, the total SW is $7400 €$ and the total TV is 200 MWh (see Fig. 17c).

In conclusion, it is clear that with the same submitted bids but different execution times, the market solutions of CT and DA are different. 